Homology, Homotopy and Applications, vol.12(1), 2010, pp.327-353

\title{
THE HORROCKS CORRESPONDENCE FOR COHERENT SHEAVES ON PROJECTIVE SPACES
}

\author{
IUSTIN COANDĂ
}

\author{
(communicated by Winfried Bruns)
}

\begin{abstract}
We establish an equivalence between the stable category of coherent sheaves (satisfying a mild restriction) on a projective space and the homotopy category of a certain class of minimal complexes of free modules over the exterior algebra Koszul dual to the homogeneous coordinate algebra of the projective space. We also relate these complexes to the Tate resolutions of the respective sheaves. In this way, we extend from vector bundles to coherent sheaves the results of G. Trautmann and the author (2005), which interpret in terms of the BGG correspondence the results of Trautmann (1978) about the correspondence of Horrocks (1964), (1977). We also give direct proofs of the BGG correspondences for graded modules and for coherent sheaves and of the theorem of Eisenbud, Fløystad and Schreyer (2003) describing the linear part of the Tate resolution associated to a coherent sheaf. Moreover, we provide an explicit description of the quotient of the Tate resolution by its linear strand corresponding to the module of global sections of the various twists of the sheaf.
\end{abstract}

\section{Introduction}

Two locally free sheaves $E$ and $E^{\prime}$ on the projective space $\mathbb{P}^{n}$ over a field $k$ are stably equivalent if there exist finite direct sums of invertible sheaves $\mathcal{O}_{\mathbb{P}}(a), a \in \mathbb{Z}, L$ and $L^{\prime}$ such that $E \oplus L \simeq E^{\prime} \oplus L^{\prime}$. Let $S=k\left[X_{0}, \ldots, X_{n}\right]$ be the homogeneous coordinate ring of $\mathbb{P}^{n} . \mathbb{P}^{n}$ being a quotient of $V \backslash\{0\}$, where $V=k^{n+1}, S$ can be identified with the symmetric algebra $S\left(V^{*}\right)$ of the dual vector space $V^{*}$. Let $\Lambda:=\bigwedge(V)$ be the exterior algebra of $V$. For $0<i<n$, the graded $S$-module $\mathrm{H}_{*}^{i} E:=\bigoplus_{d \in \mathbb{Z}} \mathrm{H}^{i}(E(d))$ is an invariant for stable equivalence. However, these cohomology $S$-modules alone do not determine uniquely the stable equivalence class of $E$. G. Horrocks [15] showed that the stable equivalence class is determined by these modules an by a sequence of extension classes. Unfortunately, the arguments of the group Ext ${ }^{1}$ in which anyone

The author was supported in part by the CNCSIS grant ID-PCE no. 51/28.09.2007 (code 304).

Received August 19, 2009; published on April 28, 2010.

2000 Mathematics Subject Classification: 14F05, 13A02, 13D25, 18 E30.

Key words and phrases: coherent sheaf, projective space, stable category, derived category.

This article is available at http://intlpress.com/HHA/v12/n1/a16

Copyright (c) 2010, International Press. Permission to copy for private use granted. 
of these extension classes lives depend on the previous extension classes. This inconvenience was removed by G. Trautmann [23] who showed that the stable equivalence class is determined by a system of matrices whose entries are (essentially) elements of the exterior algebra $\Lambda$. Trautmann's approach is related to the approach from Horrocks' paper [16].

The meaning of the matrices considered by Trautmann was clarified, following a suggestion of W. Decker, by Trautmann and the author in [9] using the BernsteinGel'fand-Gel'fand functors. These functors originate in the following easy observation: giving a linear complex of graded free $S$-modules:

$$
\cdots \longrightarrow S(p) \otimes_{k} N_{p} \longrightarrow S(p+1) \otimes_{k} N_{p+1} \longrightarrow \cdots
$$

is equivalent to giving a (left) $\Lambda$-module structure on the graded $k$-vector space $N:=\bigoplus_{p \in \mathbb{Z}} N_{p}$. One denotes the above complex by $\mathrm{F}(N)$. Similarly, to a graded $S$-module $M$ one can associate a linear complex $\mathrm{G}(M)$ of graded free $\Lambda$-modules:

$$
\cdots \longrightarrow M_{p} \otimes_{k} \bigwedge\left(V^{*}\right)(p) \longrightarrow M_{p+1} \otimes_{k} \bigwedge\left(V^{*}\right)(p+1) \longrightarrow \cdots
$$

where one considers on the exterior algebra $\bigwedge\left(V^{*}\right)$, graded such that $V^{*}$ has degree -1 , the structure of left $\Lambda$-module defined by contraction. The technical reason for which one uses $\bigwedge\left(V^{*}\right)(p)$ instead of $\Lambda(p)$ is that $\mathrm{F}\left(\bigwedge\left(V^{*}\right)\right)$ is the Koszul resolution of $S /\left(X_{0}, \ldots, X_{n}\right)$ :

$$
0 \rightarrow S(-n-1) \otimes_{k} \stackrel{n+1}{\wedge} V^{*} \rightarrow \cdots \rightarrow S(-1) \otimes_{k} V^{*} \rightarrow S \rightarrow 0
$$

The idea of I. N. Bernstein, I. M. Gel'fand and S. I. Gel'fand [4] was to extend these functors to complexes of modules by the formula $\mathrm{F}\left(N^{\bullet}\right):=\operatorname{tot}\left(X^{\bullet \bullet}\right)$, where $N^{\bullet}$ is a complex of graded $\Lambda$-modules and $X^{\bullet \bullet}$ is the double complex defined by $X^{p, \bullet}:=\mathrm{F}\left(N^{p}\right)$, and similarly for $\mathrm{G}\left(M^{\bullet}\right)$.

Now, consider the linear complex $\bigoplus_{i=1}^{n-1} \mathrm{~T}^{-i} \mathrm{G}\left(\mathrm{H}_{*}^{i} E\right.$ ) ( $\mathrm{T}$ the translation functor for complexes) with terms $G^{p}=\bigoplus_{i=1}^{n-1} \mathrm{H}^{i}(E(p-i)) \otimes_{k} \wedge\left(V^{*}\right)(p-i)$ and let $\lambda$ be its differential. The first main result of the paper $[\mathbf{9}]$ asserts that the stable equivalence class of $E$ is determined by a perturbation $d=\lambda+\delta$ of $\lambda$ obtained by addition of terms of degree $\geqslant 2$. Here "perturbation" means that $d \circ d=0$, i.e., $G^{\bullet}:=\left(\left(G^{p}\right)_{p \in \mathbb{Z}}, d\right)$ is a complex, and "obtained by addition of terms of degree $\geqslant 2$ " means that:

$$
\delta^{p}\left(\mathrm{H}^{i}(E(p-i)) \otimes_{k} \bigwedge\left(V^{*}\right)(p-i)\right) \subseteq \bigoplus_{j<i} \mathrm{H}^{j}(E(p+1-j)) \otimes_{k} \bigwedge\left(V^{*}\right)(p+1-j) .
$$

The second main result of $[\mathbf{9}]$ relates the Horrocks correspondence to the BGG correspondence via the results of Eisenbud, Fløystad and Schreyer [12] about Tate resolutions over the exterior algebra. Let $\mathcal{F}$ be a coherent sheaf on $\mathbb{P}^{n}$. Eisenbud et al. [12] show that there is a unique (up to isomorphism) perturbation of the differential of the linear complex $\bigoplus_{i=0}^{n} \mathrm{~T}^{-i} \mathrm{G}\left(\mathrm{H}_{*}^{i} \mathcal{F}\right)$ obtained by addition of terms of degree $\geqslant 2$ such that the resulting complex $I^{\bullet}$ is acyclic. It is shown in $[\mathbf{9}]$ that the complex $G^{\bullet}$ which determines the stable equivalence class of a locally free sheaf $E$ can be obtained from its Tate resolution $I^{\bullet}$ by removing the linear strands $\mathrm{G}\left(\mathrm{H}_{*}^{0} E\right)$ and $\mathrm{T}^{-n} \mathrm{G}\left(\mathrm{H}_{*}^{n} E\right)$.

In this paper, we generalize the results from $[\mathbf{9}]$ to the case of coherent sheaves using different, more natural, arguments: while in $[\mathbf{9}]$ one avoids the use of the BGG correspondence, the proofs in the present paper depend on it. In the first section we show that, using arguments close to the arguments of Horrocks [15], one can 
extend from vector bundles to coherent sheaves the splitting criterion of Horrocks and his criterion of stable equivalence. The extension to coherent sheaves of the first criterion is a result obtained recently by Abe and Yoshinaga [1] (see, also, Bertone and Roggero [5]).

In the second section we introduce and prove the properties of the BGG functors needed in the proof of the Horrocks correspondence. These are : (1) the BGG equivalence between the bounded derived category of finitely generated graded $S$-modules and the corresponding category of $\Lambda$-modules, for which we provide a direct proof, avoiding the use of Koszul duality; (2) the easy half of the Koszul duality phenomenon which says that if $N$ is a graded $\Lambda$-module then $\operatorname{GF}(N)$ is a right resolution of $N$ with graded free $\Lambda$-modules; (3) using, additionally, the functors $\operatorname{Hom}_{S}(-, S)$ for $S$ modules and $\operatorname{Hom}_{k}(-, k)$ for $\Lambda$-modules, one deduces, modulo some unpleasant sign problems, a functorial (left) free resolution for every $\Lambda$-module $N$; (4) a key technical point of the paper of Eisenbud et al. [12] describing the linear part of the minimal complex associated to a complex of free modules of the form $\mathrm{F}\left(N^{\bullet}\right)$ or $\mathrm{G}\left(M^{\bullet}\right)$. The last result is a consequence of a general lemma about double complexes, see [12, 3.5]. We explain, in Appendix A, that this lemma is a particular case of a general lemma well-known in homotopy theory under the name of Basic Perturbation Lemma.

In the third section we establish the Horrocks correspondence for coherent sheaves. It asserts that the stable category of coherent sheaves $\mathcal{F}$ on $\mathbb{P}^{n}$ with the property that $\mathrm{H}^{0} \mathcal{F}(-t)=0$ for $t \gg 0$ is equivalent to the homotopy category of minimal complexes $G^{\bullet}$ of graded free $\Lambda$-modules whose linear part is of the form $\bigoplus_{i=1}^{n-1} \mathrm{~T}^{-i} \mathrm{G}\left(H^{i}\right)$, where $H^{i}$ is the $k$-vector space graded dual of a finitely generated graded $S$-module of Krull dimension $\leqslant i+1$. This is equivalent to the fact that $G^{\bullet}$ is minimal and satisfies the following three conditions:

(i) $G^{\bullet}$ is right bounded and $\mathrm{H}^{p}\left(G^{\bullet}\right)=0$ for $p \ll 0$,

(ii) $\forall p \in \mathbb{Z}, G^{p}$ is of the form $\bigoplus_{i=1}^{n-1} \bigwedge\left(V^{*}\right)(p-i)^{c_{p i}}$,

(iii) $\lim _{p \rightarrow \infty}\left(c_{-p, i} / p^{i+1}\right)=0, i=1, \ldots, n-1$.

In the fourth section we relate the Horrocks correspondence and the BGG correspondence. We first give a direct proof of the BGG description of the bounded derived category of coherent sheaves on $\mathbb{P}^{n}$. This proof is based on an elementary comparison lemma which is discussed in Appendix B. Using the comparison lemma we also get a quick proof of the theorem of Eisenbud, Fløystad and Schreyer [12, Theorem 4.1], about Tate resolutions of coherent sheaves on $\mathbb{P}^{n}$. Moreover, we provide a concrete description of the quotient $I^{\bullet} / \mathrm{G}\left(\mathrm{H}_{*}^{0} \mathcal{F}\right)$, where $I^{\bullet}$ is the Tate resolution of a coherent sheaf $\mathcal{F}$ with $\mathrm{H}^{0}(\mathcal{F}(-t))=0$ for $t \gg 0$. Using this concrete description we derive that the complex $G^{\bullet}$ associated to $\mathcal{F}$ by the Horrocks correspondence can be obtained from its Tate resolution $I^{\bullet}$ by removing the linear strands $\mathrm{G}\left(\mathrm{H}_{*}^{0} \mathcal{F}\right)$ and $\mathrm{T}^{-n} \mathrm{G}\left(\mathrm{H}_{*}^{n} \mathcal{F}\right)$.

Notation. Throughout this paper, $V$ will denote an $(n+1)$-dimensional vector space over a field $k, e_{0}, \ldots, e_{n}$ a fixed basis of $V$ and $X_{0}, \ldots, X_{n}$ the dual basis of $V^{*}:=\operatorname{Hom}_{k}(V, k)$.

(i) Let $S=S\left(V^{*}\right)=\bigoplus_{i \geqslant 0} S^{i}\left(V^{*}\right) \simeq k\left[X_{0}, \ldots, X_{n}\right]$ be the symmetric algebra of $V^{*}$ and $S_{+}=\bigoplus_{i \geqslant 1} S^{i}\left(V^{*}\right)$ its irrelevant homogeneous ideal. We denote by $S$-Mod 
the category of graded $S$-modules with all the homogeneous components finite dimensional vector spaces, and with morphisms of degree $0 . S$-mod denotes the full subcategory of $S$-Mod consisting of finitely generated graded $S$-modules, and $\mathcal{P}$ denotes the full subcategory of $S$-mod consisting of its free objects.

(ii) If $M$ is an object of $S$-mod, the finitely generated $S$-module

$$
M^{\vee}:=\operatorname{Hom}_{S}(M, S)
$$

has a natural grading given by $\left(M^{\vee}\right)_{d}=\operatorname{Hom}_{S \text {-mod }}(M, S(d))$.

If $M$ is an object of $S$-Mod, the graded dual vector space $M^{*}$ which is, by definition, $\bigoplus_{d \in \mathbb{Z}} \operatorname{Hom}_{k}\left(M_{-d}, k\right)$ has a natural structure of graded $S$-module.

(iii) Let $\Lambda=\bigwedge(V)=\bigoplus_{i=0}^{n+1} \stackrel{i}{\wedge} V$ be the (positively graded) exterior algebra of $V$. $\Lambda_{+}:=\bigoplus_{i=1}^{n+1} \wedge$ i $V$ is an ideal of $\Lambda$. Let $\underline{k}$ denote the quotient $\Lambda / \Lambda_{+}$. We denote by $\Lambda$-mod the category of finitely generated graded left $\Lambda$-modules with morphisms of degree 0 , and by $\mathcal{I}$ its full subcategory consisting of free objects.

If $N \in \mathrm{Ob}(\Lambda-\bmod ), \operatorname{soc}(N)$ denotes the submodule of $N$ consisting of the elements annihilated by $\Lambda_{+}$. It can be identified with $\operatorname{Hom}_{\Lambda}(\underline{k}, N)$. We remark that $\operatorname{soc}(\Lambda)=\stackrel{n+1}{\wedge} V$.

(iv) Let $\mathbb{P}=\mathbb{P}^{n}=\mathbb{P}(V)$ denote the (classical) projective space parametrizing the 1-dimensional vector subspaces of $V$. The homogeneous coordinate ring of $\mathbb{P}$ is $S$. We denote by Coh $\mathbb{P}$ the category of coherent sheaves on $\mathbb{P}$ and by $(-)^{\sim}: S$-mod $\rightarrow$ Coh $\mathbb{P}$ the functor of Serre [21] associating to a graded $S$-module its sheafification. If $\mathcal{F}$ is a coherent sheaf on $\mathbb{P}$ and $0 \leqslant i \leqslant n$ we shall denote by $\mathrm{H}_{*}^{i} \mathcal{F}$ the graded $S$-module $\bigoplus_{d \in \mathbb{Z}} \mathrm{H}^{i}(\mathcal{F}(d))$.

(v) We denote by $\mathrm{C}(\mathcal{A}), \mathrm{C}^{b}(\mathcal{A}), \mathrm{C}^{ \pm}(\mathcal{A})$ the categories of complexes in an abelian category $\mathcal{A}$, by $\mathrm{K}(\mathcal{A}), \mathrm{K}^{b}(\mathcal{A}), \mathrm{K}^{ \pm}(\mathcal{A})$ the corresponding homotopy categories, and by $\mathrm{D}(\mathcal{A}), \mathrm{D}^{b}(\mathcal{A}), \mathrm{D}^{ \pm}(\mathcal{A})$ the corresponding derived categories. "T" will denote the translation functor and "Con" the mapping cone. When $\mathcal{A}$ is $S$-mod or $\Lambda$-mod or Coh $\mathbb{P}$ we shall use the shorter notation $\mathrm{C}(S), \mathrm{C}(\Lambda), \mathrm{C}(\mathbb{P})$ etc.

Our main reference for category theory will be Chapter I of the book of Kashiwara and Schapira [18]. One may also use the books of Kashiwara and Schapira [19] or Gel'fand and Manin [13].

\section{Two criteria of Horrocks}

In this section we include proofs of two introductory results which extend to coherent sheaves the splitting criterion of Horrocks for vector bundles on projective spaces and his criterion characterizing stable equivalences in the same context. There are (at least) two recently published proofs of the first result in Abe and Yoshinaga [1] and Bertone and Roggero [5]. We follow, however, Horrocks' original approach. It is based on the next theorem, which is usually proved using local cohomology and local duality (see, for example, [11, A.4.1 and A.4.2]). We shall give, for the reader's convenience, a direct proof avoiding the use of local cohomology. 
Theorem 1.1 (Graded Serre Duality). If $M$ is a finitely generated graded $S$-module then, putting $\omega_{S}:=S(-n-1) \otimes_{k} \stackrel{n+1}{\wedge} V^{*}$, there exist an exact sequence:

$$
0 \rightarrow \operatorname{Ext}_{S}^{n+1}\left(M, \omega_{S}\right)^{*} \rightarrow M \rightarrow \mathrm{H}_{*}^{0} \widetilde{M} \rightarrow \operatorname{Ext}_{S}^{n}\left(M, \omega_{S}\right)^{*} \rightarrow 0
$$

and isomorphisms: $\mathrm{H}_{*}^{i} \widetilde{M} \simeq \operatorname{Ext}_{S}^{n-i}\left(M, \omega_{S}\right)^{*}, i=1, \ldots, n$.

Proof. $\widetilde{\omega}_{S}=\mathcal{O}_{\mathbb{P}}(-n-1) \otimes_{k} \wedge^{n+1} V^{*} \simeq \omega_{\mathbb{P}}$. One knows that $\mathrm{H}^{n}\left(\omega_{\mathbb{P}}\right) \simeq k$ and that, for all $a \in \mathbb{Z}$ :

$$
\operatorname{Hom}_{\mathcal{O}_{\mathbb{P}}}\left(\mathcal{O}_{\mathbb{P}}(-a), \omega_{\mathbb{P}}\right) \stackrel{\sim}{\longrightarrow} \operatorname{Hom}_{k}\left(\mathrm{H}^{n}\left(\mathcal{O}_{\mathbb{P}}(-a)\right), \mathrm{H}^{n}\left(\omega_{\mathbb{P}}\right)\right) .
$$

It follows that if $L$ is a free graded $S$-module of finite rank then there exists a functorial isomorphism:

$$
\operatorname{Hom}_{S}\left(L, \omega_{S}\right) \stackrel{\sim}{\longrightarrow}\left(\mathrm{H}_{*}^{n} \widetilde{L}\right)^{*} .
$$

Now, let $0 \rightarrow L^{-n-1} \rightarrow \cdots \rightarrow L^{0} \rightarrow M \rightarrow 0$ be a free resolution of $M$ in $S$-mod. Let $C^{-i}$ be the cokernel of $L^{-i-1} \rightarrow L^{-i}$. One has short exact sequences:

$$
0 \rightarrow C^{-i-1} \rightarrow L^{-i} \rightarrow C^{-i} \rightarrow 0 .
$$

We consider the complex $\mathrm{H}_{*}^{n} \widetilde{L} \bullet \operatorname{Hom}_{S}\left(L^{\bullet}, \omega_{S}\right)^{*}$. Since $\mathrm{H}_{*}^{n}$ is right exact, we have exact sequences:

$$
\mathrm{H}_{*}^{n} \widetilde{L}^{-i-1} \rightarrow \mathrm{H}_{*}^{n} \widetilde{L}^{-i} \rightarrow \mathrm{H}_{*}^{n} \widetilde{C}^{-i} \rightarrow 0
$$

hence $\mathrm{H}^{0}\left(\mathrm{H}_{*}^{n} \widetilde{L} \bullet\right) \mathrm{H}_{*}^{n} \widetilde{M}$ and $\mathrm{H}^{-i}\left(\mathrm{H}_{*}^{n} \widetilde{L} \bullet\right) \operatorname{Ker}\left(\mathrm{H}_{*}^{n} \widetilde{C}^{-i} \rightarrow \mathrm{H}_{*}^{n} \widetilde{L}^{-i+1}\right)$ for $i \geqslant 1$. Since $\mathrm{H}_{*}^{p} \widetilde{L}^{-j}=0$ for $0<p<n, \forall j$, one deduces easily, using the sheafifications of the exact sequences (1), that:

$$
\mathrm{H}^{-i}\left(\mathrm{H}_{*}^{n} \widetilde{L}^{\bullet}\right) \simeq \mathrm{H}_{*}^{n-i} \widetilde{M}, \quad 0 \leqslant i \leqslant n-1
$$

and that one has exact sequences:

$$
\begin{gathered}
L^{0}=\mathrm{H}_{*}^{0} \widetilde{L}^{0} \rightarrow \mathrm{H}_{*}^{0} \widetilde{M} \rightarrow \mathrm{H}^{-n}\left(\mathrm{H}_{*}^{n} \widetilde{L}^{\bullet}\right) \rightarrow 0 \\
L^{-1}=\mathrm{H}_{*}^{0} \widetilde{L}^{-1} \rightarrow \mathrm{H}_{*}^{0} \widetilde{C}^{-1} \rightarrow \mathrm{H}^{-n-1}\left(\mathrm{H}_{*}^{n} \widetilde{L}^{\bullet}\right) \rightarrow 0,
\end{gathered}
$$

hence:

$$
\begin{gathered}
\mathrm{H}^{-n}\left(\mathrm{H}_{*}^{n} \widetilde{L}^{\bullet}\right) \simeq \operatorname{Coker}\left(M \rightarrow \mathrm{H}_{*}^{0} \widetilde{M}\right) \\
\mathrm{H}^{-n-1}\left(\mathrm{H}_{*}^{n} \widetilde{L}^{\bullet}\right) \simeq \operatorname{Coker}\left(C^{-1} \rightarrow \mathrm{H}_{*}^{0} \widetilde{C}^{-1}\right) .
\end{gathered}
$$

Finally, applying the Snake Lemma to the diagram:

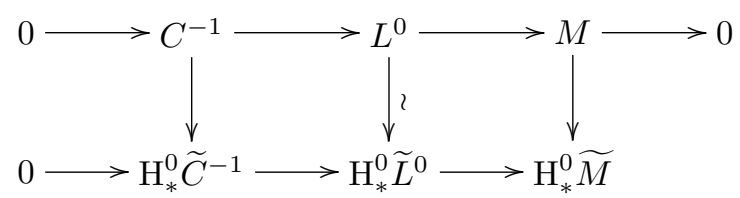

one gets that: $\operatorname{Coker}\left(C^{-1} \rightarrow \mathrm{H}_{*}^{0} \widetilde{C}^{-1}\right) \simeq \operatorname{Ker}\left(M \rightarrow \mathrm{H}_{*}^{0} \widetilde{M}\right)$.

Lemma 1.2. If $M \neq 0$ is a finitely generated graded $S$-module of projective dimension $m$ then $\operatorname{Ext}_{S}^{m}(M, S) \neq 0$. 
Proof. Let $0 \rightarrow L^{-m} \rightarrow \cdots \rightarrow L^{0} \rightarrow M \rightarrow 0$ be a free resolution of $M$ in $S$-mod. If $\operatorname{Ext}_{S}^{m}(M, S)=0$ then $L^{-m+1 \vee} \rightarrow L^{-m \vee}$ is surjective, hence its kernel $L^{\prime-m+1}$ is free and the sequence:

$$
0 \rightarrow L^{\prime-m+1} \rightarrow L^{-m+1 \vee} \rightarrow L^{-m \vee} \rightarrow 0
$$

is split exact. It follows that the dual sequence:

$$
0 \rightarrow L^{-m} \rightarrow L^{-m+1} \rightarrow\left(L^{\prime-m+1}\right)^{\vee} \rightarrow 0
$$

is exact. One gets an exact sequence:

$$
0 \rightarrow\left(L^{\prime-m+1}\right)^{\vee} \rightarrow L^{-m+2} \rightarrow \cdots \rightarrow L^{0} \rightarrow M \rightarrow 0
$$

from which we deduce that the projective dimension of $M$ is $\leqslant m-1$, a contradiction.

Theorem 1.3 (Horrocks' splitting criterion). Let $\mathcal{F}$ be a coherent sheaf on $\mathbb{P}^{n}$ with the property that $\mathrm{H}^{0}(\mathcal{F}(-t))=0$ for $t \gg 0$. If $\mathrm{H}_{*}^{i} \mathcal{F}=0$ for $0<i<n$ then $\mathcal{F}$ is a direct sum of invertible sheaves $\mathcal{O}_{\mathbb{P}}(a), a \in \mathbb{Z}$.

Proof. By hypothesis, $M:=\mathrm{H}_{*}^{0} \mathcal{F}$ is a finitely generated graded $S$-module. It follows that $M \rightarrow \mathrm{H}_{*}^{0} \widetilde{M}$ is an isomorphism. One deduces, now, from the hypothesis and from Theorem 1.1, that $\operatorname{Ext}_{S}^{i}\left(M, \omega_{S}\right)=0, \forall i>0$. It follows, from Lemma 1.2, that $M$ is a graded free $S$-module, hence a direct sum of graded $S$-modules of the form $S(a)$, $a \in \mathbb{Z}$.

Theorem 1.4 (Horrocks' criterion of stable equivalence). Let $\phi: \mathcal{F} \rightarrow \mathcal{G}$ be a morphism of coherent sheaves on $\mathbb{P}^{n}, n \geqslant 2$, with the property that $\mathrm{H}^{0} \phi(-t)$ is an isomorphism for $t \gg 0$. If $\mathrm{H}_{*}^{i} \phi$ is an isomorphism for $0<i<n$ then $\phi$ factorizes as:

$$
\mathcal{F} \hookrightarrow \mathcal{F} \oplus \mathcal{A} \stackrel{\sim}{\longrightarrow} \oplus \mathcal{G} \rightarrow \mathcal{G}
$$

where the first morphism is the canonical inclusion, $\mathcal{A}$ and $\mathcal{B}$ are finite direct sums of invertible sheaves $\mathcal{O}_{\mathbb{P}}(a), a \in \mathbb{Z}$, and the last morphism is the canonical projection.

Proof. Choose $m \in \mathbb{Z}$ such that $\mathrm{H}^{0} \phi(-t)$ is an isomorphism for $t>m$ and let $M:=\bigoplus_{j \geqslant-m} \mathrm{H}^{0}(\mathcal{F}(j)), N:=\bigoplus_{j \geqslant-m} \mathrm{H}^{0}(\mathcal{G}(j))$. Choose an epimorphism $g: A \rightarrow N$, with $A$ a finitely generated graded free $S$-module. Let $\pi: \mathcal{F} \oplus \widetilde{A} \rightarrow \mathcal{G}$ be the epimorphism defined by $\phi$ and $\widetilde{g}$ and let $\mathcal{B}$ be the kernel of $\pi$. Using the exact sequence:

$$
0 \rightarrow \mathcal{B} \rightarrow \mathcal{F} \oplus \widetilde{A} \rightarrow \mathcal{G} \rightarrow 0
$$

one sees that $\mathcal{B}$ satisfies the hypothesis of Theorem 1.3 , hence $\mathcal{B}$ is a direct sum of invertible sheaves $\mathcal{O}_{\mathbb{P}}(b), b \in \mathbb{Z}$, and, consequently, $B:=\mathrm{H}_{*}^{0} \mathcal{B}$ is a graded free $S$-module. Applying $\mathrm{H}_{*}^{0}$ to the exact sequence $\left(^{*}\right)$ and cancelling the isomorphism $\bigoplus_{j<-m} \mathrm{H}^{0} \mathcal{F}(j) \stackrel{\sim}{\rightarrow} \bigoplus_{j<-m} \mathrm{H}^{0} \mathcal{G}(j)$ one gets a short exact sequence:

$$
0 \rightarrow B \rightarrow M \oplus A \rightarrow N \rightarrow 0 .
$$

Since $\mathrm{H}_{*}^{n-1} \phi$ is an isomorphism, it follows from Theorem 1.1 that:

$$
\operatorname{Ext}_{S}^{1}(N, B) \longrightarrow \operatorname{Ext}_{S}^{1}(M \oplus A, B)
$$

is an isomorphism, hence $\operatorname{Hom}_{S}(M \oplus A, B) \rightarrow \operatorname{Hom}_{S}(B, B)$ is surjective, hence the exact sequence $(* *)$ splits. 


\section{The BGG functors}

Definition 2.1. When dealing with the category $\Lambda$-mod one encounters sign problems. In order to avoid any complication we shall observe strictly the Koszul sign convention (when two homogeneous symbols $\xi$ and $\eta$ are permuted the result is multiplied by $\left.(-1)^{\operatorname{deg} \xi \cdot \operatorname{deg} \eta}\right)$.

(i) If $K, N \in \mathrm{Ob}\left(\Lambda\right.$-mod) the graded $k$-vector space $K \otimes_{k} N$ has a structure of left $\Lambda$-module given by:

$$
v \cdot(x \otimes y):=(v \cdot x) \otimes y+(-1)^{\operatorname{deg} x} x \otimes(v \cdot y), \quad \text { for } v \in V .
$$

In particular, we put $N(a):=\underline{k}(a) \otimes_{k} N$, for $a \in \mathbb{Z}$. The grading of $N(a)$ is given by $N(a)_{p}=N_{p+a}$ and the $\Lambda$-module structure by: $(v \cdot y)_{N(a)}=(-1)^{a}(v \cdot y)_{N}$, for $v \in V, y \in N$. With this definition, if $v \in V$ then the morphism of $k$-vector spaces $(v \cdot-)_{N}: N \rightarrow N$ is a morphism in $\Lambda$-mod: $N(a) \rightarrow N(a+1), \forall a \in \mathbb{Z}$. If $\phi: K \rightarrow N$ is a morphism in $\Lambda$-mod, $\phi(a): K(a) \rightarrow N(a)$ is just $\phi$ if one forgets the gradings. However, if $N^{\bullet} \in \mathrm{ObC}\left(\Lambda\right.$-mod) then $N^{\bullet}(a)$ is, by definition, the complex with terms $\left(N^{p}(a)\right)_{p \in \mathbb{Z}}$ but with $d_{N(a)}:=(-1)^{a} d_{N}$ (the differential of a complex is a symbol of degree 1 !).

(ii) If $K, N \in \mathrm{Ob}\left(\Lambda\right.$-mod) the graded $k$-vector space $\operatorname{Hom}_{k}(N, K)$ has a structure of left $\Lambda$-module given by:

$$
(v \cdot \phi)(y):=v \cdot \phi(y)-(-1)^{\operatorname{deg} \phi} \phi(v \cdot y), \quad \text { for } v \in V .
$$

In particular, for $K=\underline{k}$, one puts $N^{*}:=\operatorname{Hom}_{k}(N, \underline{k})$. One has $\left(N^{*}\right)_{p}=\left(N_{-p}\right)^{*}$, and for $v \in V,(v \cdot-)_{N^{*}}:\left(N^{*}\right)_{p} \rightarrow\left(N^{*}\right)_{p+1}$ is $(-1)^{p+1}$ times the dual of $(v \cdot-)_{N}$ : $N_{-p-1} \rightarrow N_{-p}$.

The map $\mu: N \rightarrow N^{* *}, \mu(y)(\phi):=(-1)^{\operatorname{deg} y \cdot \operatorname{deg} \phi} \phi(y) \quad$ (i.e., with $\mu_{p}:=(-1)^{p}$ can: $\left.N_{p} \rightarrow\left(N_{p}\right)^{* *}, p \in \mathbb{Z}\right)$ is an isomorphism in $\Lambda$-mod.

(iii) The map $\alpha: K^{*} \otimes_{k} N^{*} \rightarrow\left(K \otimes_{k} N\right)^{*}$ given by:

$$
\alpha(f \otimes g)(x \otimes y):=(-1)^{\operatorname{deg} g \cdot \operatorname{deg} x} f(x) g(y)
$$

is an isomorphism in $\Lambda$-mod. In particular, for $a \in \mathbb{Z}$, one gets an isomorphism in $\Lambda$-mod $\alpha: N^{*}(-a) \stackrel{\sim}{\rightarrow}(N(a))^{*}$ with $\alpha_{p}=(-1)^{(p-a) a} \operatorname{id}_{\left(N_{a-p}\right)^{*}}, p \in \mathbb{Z}$.

Under these identifications, if $v \in V$ and $a \in \mathbb{Z}$, the dual of the morphism $(v \cdot-)_{N}: N(-a-1) \rightarrow N(-a)$ is identified with the morphism

$$
(-1)^{a}(v \cdot-)_{N^{*}}: N^{*}(a) \rightarrow N^{*}(a+1) .
$$

(iv) We endow $\bigwedge\left(V^{*}\right)=\bigoplus_{i=0}^{n+1} \stackrel{i}{\wedge} V^{*}$, graded such that $\stackrel{i}{\wedge} V^{*}=\bigwedge\left(V^{*}\right)_{-i}$, with the structure of graded left $\Lambda$-module given by:

$$
v \cdot f_{1} \wedge \ldots \wedge f_{p}:=\sum_{i=1}^{p}(-1)^{i-1} f_{i}(v) f_{1} \wedge \ldots \wedge \widehat{f}_{i} \wedge \ldots \wedge f_{p}, \quad v \in V, f_{1}, \ldots, f_{p} \in V^{*} .
$$

The unique morphism of left $\Lambda$-modules $\Lambda \rightarrow \bigwedge\left(V^{*}\right)^{*}$ (resp., $\Lambda \rightarrow \bigwedge\left(V^{*}\right)(-n-1) \otimes_{k}$ $\left.{ }^{n+1} V\right)$ mapping $1 \in \Lambda_{0}$ to $1 \in\left(\Lambda\left(V^{*}\right)^{*}\right)_{0}$ (respectively, to the element $\operatorname{id}_{n+1}$ of $\left.\left(\bigwedge\left(V^{*}\right)(-n-1) \otimes_{k} \stackrel{n+1}{\wedge} V\right)_{0}\right)$ is an isomorphism in $\Lambda$-mod. 
The following lemma, whose standard proof can be found, for example, in [8, 4(i)], which shows in particular that $\forall a \in \mathbb{Z}, \bigwedge\left(V^{*}\right)(a)$ is an injective object of $\Lambda$-mod.

Lemma 2.2. $\forall N \in \mathrm{Ob}(\Lambda$-mod $), \forall a \in \mathbb{Z}$, the map:

$$
\operatorname{Hom}_{\Lambda-\bmod }\left(N, \bigwedge\left(V^{*}\right)(a)\right) \longrightarrow\left(N_{-a}\right)^{*}, \quad \phi \mapsto \phi_{-a}
$$

is bijective.

Corollary 2.3. $\phi_{-a-1}: N_{-a-1} \rightarrow \bigwedge\left(V^{*}\right)(a)_{-a-1}=V^{*}$ can be described by:

$$
\phi_{-a-1}(y)(v)=(-1)^{a} \phi_{-a}(v \cdot y), \quad \forall y \in N_{-a-1}, \forall v \in V,
$$

or, equivalently, by: $\phi_{-a-1}=(-1)^{a} \sum_{i=0}^{n}\left(\phi_{-a} \circ\left(e_{i} \cdot-\right)_{N}\right) \otimes X_{i}$.

Proof. Recalling the Definition 2.1(i), (ii), one has, for $v \in V, \lambda \in V^{*}$ :

$$
(v \cdot \lambda)_{\wedge\left(V^{*}\right)(a)}=(-1)^{a}(v \cdot \lambda) \wedge\left(V^{*}\right)=(-1)^{a} \lambda(v) .
$$

In particular, for $\lambda=\phi_{-a-1}(y)$, since $\phi$ is a morphism in $\Lambda$-mod:

$$
(-1)^{a} \phi_{-a-1}(y)(v)=\left(v \cdot \phi_{-a-1}(y)\right)_{\wedge\left(V^{*}\right)(a)}=\phi_{-a}(v \cdot y) .
$$

Definition 2.4. (The BGG functors)

(i) One defines a functor $\mathrm{F}: \Lambda$-mod $\rightarrow \mathrm{C}^{b}(\mathcal{P})$ by: $\mathrm{F}(N)^{p}:=S(p) \otimes_{k} N_{p}, d_{\mathrm{F}(N)}:=$ $\sum_{i=0}^{n}\left(X_{i} \cdot-\right)_{S} \otimes\left(e_{i} \cdot-\right)_{N}$. F can be extended to a functor $\mathrm{F}: \mathrm{C}^{b}(\Lambda$-mod $) \rightarrow \mathrm{C}^{b}(\mathcal{P})$ by putting $\mathrm{F}\left(N^{\bullet}\right):=\operatorname{tot}\left(X^{\bullet \bullet}\right)$, where $X^{\bullet \bullet}$ is the double complex with $X^{p, \bullet}:=\mathrm{F}\left(N^{p}\right)$ and with $d_{X}^{\prime p}: X^{p, \bullet} \rightarrow X^{p+1, \bullet}$ equal to $\mathrm{F}\left(d_{N}^{p}\right)$.

(ii) One defines a functor $\mathrm{G}: S$-Mod $\rightarrow \mathrm{C}(\mathcal{I})$ by: $\mathrm{G}(M)^{p}:=M_{p} \otimes_{k} \bigwedge\left(V^{*}\right)(p)$ and $d_{\mathrm{G}(M)}:=\sum_{i=0}^{n}\left(X_{i} \cdot-\right)_{M} \otimes\left(e_{i} \cdot-\right) \wedge\left(V^{*}\right) \cdot \mathrm{G}$ can be extended, in a similar way, to a functor $\mathrm{G}: \mathrm{C}^{b}(S$-Mod $) \rightarrow \mathrm{C}(\mathcal{I})$. The (extended) functor $\mathrm{G}$ maps $\mathrm{C}^{b}(S$-mod) to $\mathrm{C}^{+}(\mathcal{I})$.

(iii) $\mathrm{F}$ and $\mathrm{G}$ commute with the translation functors and with mapping cones.

Definition 2.5. Let $\Phi: \mathcal{A}^{\mathrm{op}} \rightarrow \mathcal{B}$ be an additive contravariant functor between two additive categories $\mathcal{A}$ and $\mathcal{B}$. If $X^{\bullet} \in \mathrm{ObC}(\mathcal{A})$ one defines a complex $\Phi\left(X^{\bullet}\right) \in \mathrm{Ob} \mathrm{C}(\mathcal{B})$ by:

$$
\Phi\left(X^{\bullet}\right)^{p}:=\Phi\left(X^{-p}\right), \quad d_{\Phi(X)}^{p}:=(-1)^{p+1} \Phi\left(d_{X}^{-p-1}\right): \Phi\left(X^{-p}\right) \rightarrow \Phi\left(X^{-p-1}\right) .
$$

For example, if $M^{\bullet} \in \mathrm{Ob} \mathrm{C}(S$-mod $)$ one can define the complex $M^{\bullet \vee}$ in $\mathrm{C}(S$-mod $)$ and if $M^{\bullet} \in \mathrm{ObC}\left(S\right.$-Mod) (resp., $N^{\bullet} \in \mathrm{ObC}(\Lambda$-mod)) one can define the complex $M^{\bullet *} \in \mathrm{ObC}\left(S\right.$-Mod) (resp., $N^{\bullet *} \in \mathrm{ObC}(\Lambda$-mod) $)$.

Furthermore, if $X^{\bullet \bullet}$ is a double complex in $\mathcal{A}$ one defines a double complex $\Phi\left(X^{\bullet \bullet}\right)$ in $\mathcal{B}$ by $\Phi\left(X^{\bullet \bullet}\right)^{p q}:=\Phi\left(X^{-p,-q}\right)$ and

$$
d_{\Phi(X)}^{\prime p q}:=(-1)^{p+1} \Phi\left(d_{X}^{\prime-p-1,-q}\right), \quad d_{\Phi(X)}^{\prime \prime p q}:=(-1)^{q+1} \Phi\left(d_{X}^{\prime \prime-p,-q-1}\right) .
$$

If we denote $\Phi\left(X^{\bullet \bullet}\right)$ by $Y^{\bullet \bullet}$ then $Y^{p, \bullet}=\Phi\left(X^{-p, \bullet}\right)$ and $d_{Y}^{\prime p, \bullet}=(-1)^{p+1} \Phi\left(d_{X}^{\prime-p-1, \bullet}\right)$.

Lemma 2.6. (a) If $X^{\bullet \bullet}$ and $Y^{\bullet \bullet}$ are two double complexes with $X^{p q}=Y^{p q}, \forall p, q$, but with $d_{Y}^{\prime}=(-1)^{a} d_{X}^{\prime}$ and $d_{Y}^{\prime \prime}=(-1)^{b} d_{X}^{\prime \prime}$, for some $a, b \in \mathbb{Z}$, then $X \bullet \simeq Y^{\bullet \bullet}$.

(b) Using the notations from the last part of Definition 2.5, assume that, $\forall m \in \mathbb{Z}$, the set $\left\{(p, q) \mid p+q=m, \quad X^{p q} \neq 0\right\}$ is finite. Then $\operatorname{tot}\left(\Phi\left(X^{\bullet \bullet}\right)\right) \simeq \Phi\left(\operatorname{tot}\left(X^{\bullet \bullet}\right)\right)$. 
Proof. (a) $\left((-1)^{a p+b q} \mathrm{id}_{X p q}\right)_{p, q \in \mathbb{Z}}$ is an isomorphism of double complexes $X^{\bullet \bullet} \stackrel{\sim}{\rightarrow} Y^{\bullet \bullet}$.

(b) One can easily check that $\Phi\left(\operatorname{tot}\left(X^{\bullet \bullet}\right)\right)=\operatorname{tot}\left(Z^{\bullet \bullet}\right)$, where the double complex $Z^{\bullet \bullet}$ is defined by:

$Z^{p q}:=\Phi\left(X^{-p-q}\right), d_{Z}^{p q}:=(-1)^{p+q+1} \Phi\left(d_{X}^{\prime-p-1,-q}\right), d_{Z}^{\prime \prime p q}:=(-1)^{p+q+1} \Phi\left(d_{X}^{\prime \prime-p,-q-1}\right)$.

But $\left((-1)^{p q} \operatorname{id}_{\Phi\left(X^{-p,-q}\right)}\right)_{p, q \in \mathbb{Z}}$ is an isomorphism of double complexes $\Phi\left(X^{\bullet \bullet}\right) \stackrel{\sim}{\rightarrow} Z \bullet$.

Lemma 2.7. Let $M^{\bullet} \in \mathrm{ObC}^{b}(S-\mathrm{Mod}), N^{\bullet} \in \mathrm{ObC}^{b}(\Lambda$-mod $)$ and $a \in \mathbb{Z}$. Then:

(a) $\mathrm{F}\left(N^{\bullet}(a)\right)=\mathrm{T}^{a} \mathrm{~F}\left(N^{\bullet}\right)(-a)$ and $\mathrm{G}\left(M^{\bullet}(a)\right)=\mathrm{T}^{a} \mathrm{G}\left(M^{\bullet}\right)(-a)$ (one has equality, not only an isomorphism!),

(b) $\mathrm{F}\left(N^{\bullet *}\right) \simeq \mathrm{F}\left(N^{\bullet}\right)^{\vee}$,

(c) $\mathrm{G}\left(M^{\bullet}\right)^{*} \simeq \mathrm{G}\left(M^{\bullet *}\right)(-n-1) \otimes_{k} \wedge^{n+1} V \simeq \mathrm{T}^{-n-1} \mathrm{G}\left(\left(M^{\bullet} \otimes_{S} \omega_{S}\right)^{*}\right)$.

Proof. (a) One checks, firstly, that if $M \in \mathrm{Ob}(S$-Mod) and $N \in \mathrm{Ob}(\Lambda$-mod) then $\mathrm{F}(N)=\mathrm{T}^{a} \mathrm{~F}(N)(-a)$ and $\mathrm{G}(M)=\mathrm{T}^{a} \mathrm{G}(M)(-a)$. For the general case, one takes into account the sign convention at the end of Definition 2.1(i).

(b) If $N \in \mathrm{Ob}\left(\Lambda\right.$-mod) then one checks easily that $\mathrm{F}\left(N^{*}\right)=\mathrm{F}(N)^{\vee}$. Now, if $N^{\bullet} \in$ $\mathrm{Ob} \mathrm{C}^{b}(\Lambda$-mod $)$ then, by defintion, $\mathrm{F}\left(N^{\bullet}\right)=\operatorname{tot}\left(X^{\bullet \bullet}\right)$ with $X^{p, \bullet}=\mathrm{F}\left(N^{p}\right), \forall p \in \mathbb{Z}$. One deduces that, using the last part of Definition $2.5, \mathrm{~F}\left(N^{\bullet *}\right)=\operatorname{tot}\left(\left(X^{\bullet \bullet}\right)^{\vee}\right)$. But, by Lemma $2.6(\mathrm{~b}), \operatorname{tot}\left(\left(X^{\bullet \bullet}\right)^{\vee}\right) \simeq \operatorname{tot}\left(X^{\bullet \bullet}\right)^{\vee}$.

(c) If $N \in \mathrm{Ob}\left(\Lambda\right.$-mod), one can define a functor $\mathrm{G}_{N}: S$-Mod $\rightarrow \mathrm{C}(\Lambda$-mod) by $\mathrm{G}_{N}(M)^{p}:=M_{p} \otimes_{k} N(p), d_{\mathrm{G}_{N}(M)}:=\sum_{i=0}^{n}\left(X_{i} \cdot-\right)_{M} \otimes\left(e_{i} \cdot-\right)_{N}$. As in Definition $2.4, \mathrm{G}_{N}$ can be extended to a functor $\mathrm{G}_{N}: \mathrm{C}^{b}(S-\operatorname{Mod}) \rightarrow \mathrm{C}(\Lambda$-mod $)$.

First, if $M \in \mathrm{Ob}\left(S\right.$-Mod) then, by Definition $2.1(\mathrm{iii}), \mathrm{G}_{N^{*}}\left(M^{*}\right)^{p} \stackrel{\sim}{\rightarrow}\left(\mathrm{G}_{N}(M)^{-p}\right)^{*}$, $\forall p \in \mathbb{Z}$, and under these identifications, $d_{\mathrm{G}_{N^{*}}\left(M^{*}\right)}$ is identified with $(-1)^{p}\left(d_{\mathrm{G}_{N}(M)}^{-p-1}\right)^{*}$. Recalling the Definition 2.5, it follows that $\mathrm{G}_{N^{*}}\left(M^{*}\right)$ is isomorphic to a complex whose terms coincide with the terms of $\mathrm{G}_{N}(M)^{*}$ but whose differential equals "_." the differential of $\mathrm{G}_{N}(M)^{*}$. Using Lemma 2.6, one deduces now, for every complex $M^{\bullet} \in \mathrm{ObC}^{b}(S$-Mod $)$, an isomorphism $\mathrm{G}_{N^{*}}\left(M^{\bullet *}\right) \stackrel{\sim}{\rightarrow} \mathrm{G}_{N}\left(M^{\bullet}\right)^{*}$.

Secondly, if $M \in \mathrm{Ob}(S$-Mod) and $a \in \mathbb{Z}$ then, taking into account the sign convention at the end of Definition 2.1(i), $\mathrm{G}_{N(a)}(M)=\mathrm{G}_{N}(M)(a)$. Using Lemma 2.6(a) one deduces, for every complex $M^{\bullet} \in \mathrm{ObC}^{b}(S$-Mod $)$, an isomorphism $\mathrm{G}_{N(a)}\left(M^{\bullet}\right) \simeq$ $\mathrm{G}_{N}\left(M^{\bullet}\right)(a)$.

Since $\mathrm{G}:=\mathrm{G}_{\wedge\left(V^{*}\right)}$ one gets, recalling the isomorphisms at the end of Definition 2.1(iv), the first isomorphism from the statement. The second isomorphism follows from (a).

Definition 2.8. (The linear part of a minimal complex)

(i) Let $L^{\bullet} \in \mathrm{ObC}(\mathcal{P})$. One may write $L^{i}=\bigoplus_{j \in \mathbb{Z}} S(i-j) b_{i j}$. For $m \in \mathbb{Z}$ one puts:

$$
F_{m} L^{i}:=\bigoplus_{j \leqslant m} S(i-j)^{b_{i j}}
$$

Alternatively, $F_{m} L^{i}$ is the $S$-submodule of $L^{i}$ generated by the homogeneous elements of degree $\leqslant m-i$. The complex $L^{\bullet}$ is called minimal if $\operatorname{Im} d_{L} \subseteq S_{+} \cdot L^{\bullet}$. This is equivalent to the fact, $\forall m \in \mathbb{Z}, F_{m} L^{\bullet}:=\left(F_{m} L^{i}\right)_{i \in \mathbb{Z}}$ is a subcomplex of $L^{\bullet}$. In this case, $\operatorname{gr}_{F}\left(L^{\bullet}\right)$ is called the linear part of $L^{\bullet}$. 
(ii) Similarly, let $I^{\bullet} \in \mathrm{ObC}(\mathcal{I})$. One may write (by the last part of Definition 2.1(iv)) $I^{i}=\bigoplus_{j \in \mathbb{Z}} \bigwedge\left(V^{*}\right)(i-j){ }^{c_{i j}}$. For $m \in \mathbb{Z}$, one puts:

$$
F_{m} I^{i}:=\bigoplus_{j \leqslant m} \wedge\left(V^{*}\right)(i-j)^{c_{i j}} .
$$

Alternatively, $F_{m} I^{i}$ is the $\Lambda$-submodule of $I^{i}$ generated by the homogeneous elements of degree $\leqslant m-i-n-1$. The complex $I^{\bullet}$ is called minimal if $\operatorname{Im} d_{I} \subseteq \Lambda_{+} \cdot I^{\bullet}$ or, equivalently, if $F_{m} I^{\bullet}:=\left(F_{m} I^{i}\right)_{i \in \mathbb{Z}}$ is a subcomplex of $I^{\bullet}, \forall m \in \mathbb{Z}$. In this case, $\operatorname{gr}_{F}\left(I^{\bullet}\right)$ is called the linear part of $I^{\bullet}$.

(iii) If two minimal complexes from $\mathrm{C}(\mathcal{I})$ are isomorphic in $\mathrm{K}(\mathcal{I})$ (i.e., are homotopically equivalent) then they are isomorphic in $\mathrm{C}(\mathcal{I})$ (see, for example, $[\mathbf{9}, 4.2]$ ).

The following result, which is a direct consequence of Lemma A.7 from Appendix A, is one of the key points of the paper of Eisenbud, Fløystad, and Schreyer [12].

Lemma 2.9. (a) If $N^{\bullet} \in \mathrm{ObC}^{b}\left(\Lambda\right.$-mod) then $\mathrm{F}\left(N^{\bullet}\right) \in \mathrm{ObC}^{b}(\mathcal{P})$ can be contracted to a minimal complex $L^{\bullet}$ whose linear part is $\mathrm{F}\left(\mathrm{H}^{\bullet}\left(N^{\bullet}\right)\right)$, where $\mathrm{H}^{\bullet}\left(N^{\bullet}\right)$ is the complex with terms $\mathrm{H}^{p}\left(N^{\bullet}\right), p \in \mathbb{Z}$, and with the differential equal to 0 . Moreover, this contraction induces, $\forall m \in \mathbb{Z}$, a contraction of $\mathrm{F}\left(\tau^{\leqslant m} N^{\bullet}\right)$ onto $F_{m} L^{\bullet}$ and of $\mathrm{F}\left(\tau^{>m} N^{\bullet}\right)$ onto $L^{\bullet} / F_{m} L^{\bullet}$.

(b) If $M^{\bullet} \in \mathrm{Ob} \mathrm{C}^{b}\left(S\right.$-Mod) then $\mathrm{G}\left(M^{\bullet}\right) \in \mathrm{ObC}(\mathcal{I})$ can be contracted to a minimal complex $I^{\bullet}$ whose linear part is $\mathrm{G}\left(\mathrm{H}^{\bullet}\left(M^{\bullet}\right)\right)$. Moreover, this contraction induces a contraction of $\mathrm{G}\left(\tau^{\leqslant m} M^{\bullet}\right)$ onto $F_{m} I^{\bullet}$ and of $\mathrm{G}\left(\tau^{>m} M^{\bullet}\right)$ onto $I^{\bullet} / F_{m} I^{\bullet}, \forall m \in \mathbb{Z}$.

The next theorem is the Bernstein-Gel'fand-Gel'fand correspondence for graded modules. We include here a direct proof, which does not use Koszul duality. We use, instead, the Comparison Lemma B.1 from Appendix B.

Theorem $2.10\left(\left[\mathbf{4}\right.\right.$, Theorem 3]). The functor $\mathrm{F}: \mathrm{C}^{b}(\Lambda$-mod $) \rightarrow \mathrm{C}^{b}(\mathcal{P})$ extends to an equivalence of triangulated categories $\mathrm{F}: \mathrm{D}^{b}(\Lambda$-mod $) \rightarrow \mathrm{K}^{b}(\mathcal{P})$.

Proof. If $\phi: N^{\bullet \bullet} \rightarrow N^{\bullet}$ is a quasi-isomorphism in $\mathrm{C}^{b}(\Lambda$-mod) then $\operatorname{Con}(\phi)$ is acyclic. By Lemma 2.9(a), the complex $\operatorname{Con}(\mathrm{F}(\phi))=\mathrm{F}(\operatorname{Con}(\phi))$ is homotopically equivalent to 0 , whence $\mathrm{F}(\phi)$ is a homotopic equivalence. One deduces that $\mathrm{F}$ extends to a functor $\mathrm{F}: \mathrm{D}^{b}(\Lambda$-mod $) \rightarrow \mathrm{K}^{b}(\mathcal{P})$.

We show, firstly, that this functor is fully faithful, i.e., that if $N^{\bullet}, N^{\prime \bullet}$ are two complexes from $\mathrm{C}^{b}(\Lambda$-mod) then:

$$
\operatorname{Hom}_{\mathrm{D}^{b}(\Lambda)}\left(N^{\bullet}, N^{\bullet}\right) \stackrel{\sim}{\longrightarrow} \operatorname{Hom}_{\mathrm{K}^{b}(\mathcal{P})}\left(\mathrm{F}\left(N^{\bullet \bullet}\right), \mathrm{F}\left(N^{\bullet}\right)\right) .
$$

We endow $N^{\bullet}$ and $N^{\prime \bullet}$ with the filtrations $F^{i} N^{\bullet}=\sigma^{\geqslant i} N^{\bullet}, F^{i} N^{\bullet}=\sigma^{\geqslant i} N^{\prime \bullet}$ with successive quotients $\mathrm{T}^{-i} N^{i}$ and $\mathrm{T}^{-i} N^{\prime i}$, respectively. If, $\forall i, j \in \mathbb{Z}$, the map:

$$
\operatorname{Hom}_{\mathrm{D}^{b}(\Lambda)}\left(N^{\prime i}, \mathrm{~T}^{p} N^{j}\right) \longrightarrow \operatorname{Hom}_{\mathrm{K}^{b}(\mathcal{P})}\left(\mathrm{F}\left(N^{\prime i}\right), \mathrm{F}\left(\mathrm{T}^{p} N^{j}\right)\right)
$$

would be bijective for $i-j-1 \leqslant p \leqslant i-j+1$ then Lemma B.1, applied to the functor $\mathrm{F}: \mathrm{D}^{b}(\Lambda$-mod $) \rightarrow \mathrm{K}^{b}(\mathcal{P})$, would imply that $\left(^{*}\right)$ is bijective. Now, if $K$ and $K^{\prime}$ are two objects of $\Lambda$-mod then:

$$
\operatorname{Hom}_{\mathrm{D}^{b}(\Lambda)}\left(K^{\prime}, K\right) \stackrel{\sim}{\longrightarrow} \operatorname{Hom}_{\mathrm{K}^{b}(\mathcal{P})}\left(\mathrm{F}\left(K^{\prime}\right), \mathrm{F}(K)\right)
$$

as one can easily see using the fact that $\operatorname{Hom}_{\mathrm{D}^{b}(\Lambda)}\left(K^{\prime}, K\right) \simeq \operatorname{Hom}_{\Lambda \text {-mod }}\left(K^{\prime}, K\right)$. Moreover, $\operatorname{Hom}_{\mathrm{D}^{b}(\Lambda)}\left(K^{\prime}, \mathrm{T}^{p} K\right)=0$ for $p<0$ (see B.3) and $\operatorname{Hom}_{\mathrm{D}^{b}(\Lambda)}\left(K^{\prime}, \mathrm{T}^{p} K\right)=0$ for 
$p>0$ if $K$ is a direct sum of $\Lambda$-modules of the form $\Lambda\left(V^{*}\right)(a)$ because, in this case, $K$ is an injective object of $\Lambda$-mod.

On the other hand, $\operatorname{Hom}_{\mathrm{K}^{b}(\mathcal{P})}\left(\mathrm{F}\left(K^{\prime}\right), \mathrm{F}\left(\mathrm{T}^{p} K\right)\right)=0$ for $p<0$ because $\mathrm{F}\left(K^{\prime}\right)^{i}=$ $S(i) \otimes_{k} K_{i}^{\prime}$ and $\mathrm{F}\left(\mathrm{T}^{p} K\right)^{i}=\mathrm{F}(K)^{i+p}=S(i+p) \otimes_{k} K_{i+p}, \quad \forall i \in \mathbb{Z}$. Moreover, if $L^{\bullet} \in \operatorname{ObC}^{b}(\mathcal{P})$ and $\mathrm{H}^{i}\left(L^{\bullet}\right)_{-i}=0, \forall i \in \mathbb{Z}$, then $\operatorname{Hom}_{\mathrm{K}^{b}(\mathcal{P})}\left(\mathrm{F}\left(K^{\prime}\right), L^{\bullet}\right)=0$ because

$$
\operatorname{Hom}_{\mathrm{K}^{b}(\mathcal{P})}\left(\mathrm{T}^{-i} \mathrm{~F}\left(K^{\prime}\right)^{i}, L^{\bullet}\right) \simeq \mathrm{H}^{i}\left(L^{\bullet}\right)_{-i} \otimes_{k}\left(K_{i}^{\prime}\right)^{*}=0, \quad \forall i \in \mathbb{Z},
$$

and $\mathrm{F}\left(K^{\prime}\right)$ can be endowed with the filtration $\sigma^{\geqslant i} \mathrm{~F}\left(K^{\prime}\right), i \in \mathbb{Z}$, with successive quotients $\mathrm{T}^{-i} \mathrm{~F}\left(K^{\prime}\right)^{i}$. For $L^{\bullet}=\mathrm{F}\left(\mathrm{T}^{p} K\right)$, the condition $\mathrm{H}^{i}\left(L^{\bullet}\right)_{-i}=0, \forall i \in \mathbb{Z}$, is fulfilled if $p>0$ and $K$ is a direct sum of $\Lambda$-modules of the form $\Lambda\left(V^{*}\right)(a)$, because $\mathrm{F}\left(\mathrm{T}^{p} \bigwedge\left(V^{*}\right)(a)\right)=\mathrm{T}^{p+a} \mathrm{~F}\left(\bigwedge\left(V^{*}\right)\right)(-a) \& \mathrm{~F}\left(\bigwedge\left(V^{*}\right)\right)$ is the Koszul resolution of $S / S_{+}$.

Summing up, if $N^{j}$ is a direct sum of $\Lambda$-modules of the form $\Lambda\left(V^{*}\right)(a)$, $\forall j \leqslant \sup \left\{i \in \mathbb{Z} \mid N^{\prime i} \neq 0\right\}$, then Lemma B.1 implies that $(*)$ is bijective. If $N^{\bullet}$ is arbitrary, one constructs, using Lemma 2.2 , a quasi-isomorphism $N^{\bullet} \rightarrow I^{\bullet}$ with $I^{\bullet} \in \mathrm{ObC}^{+}(\mathcal{I})$. For $m>\sup \left\{i \in \mathbb{Z} \mid N^{\prime i} \neq 0\right\}$ large enough, one gets a quasi-isomorphism $N^{\bullet} \rightarrow \tau^{\leqslant m} I^{\bullet}$. By what has been proved, $\left(^{*}\right)$ is bijective for the pair $\left(N^{\bullet \bullet}, \tau^{\leqslant m} I^{\bullet}\right)$, hence also for the pair $\left(N^{\prime \bullet}, N^{\bullet}\right)$.

Finally, the essential surjectivity can be checked as follows. By what has been proved, the image of $\mathrm{F}: \mathrm{D}^{b}(\Lambda$-mod $) \rightarrow \mathrm{K}^{b}(\mathcal{P})$ is a full subcategory of $\mathrm{K}^{b}(\mathcal{P})$, closed under the functors $\mathrm{T}$ and $\mathrm{T}^{-1}$ and under mapping cones. Moreover, $\mathrm{F}\left(\mathrm{T}^{a} \underline{k}(-a)\right)=$ $S(a), \forall a \in \mathbb{Z}$. If $L^{\bullet} \in \mathrm{ObK}^{b}(\mathcal{P})$ one deduces easily, by induction on $\sum_{i \in \mathbb{Z}} \mathrm{rk} L^{i}$, that $L^{\bullet}$ is isomorphic in $\mathrm{K}^{b}(\mathcal{P})$ to a complex in the image of $\mathrm{F}$.

Actually, the authors of [4] prove something more, namely that one can get a quasiinverse to $\mathrm{F}$ by applying $\mathrm{G}$ and then taking convenient truncations (see Beilinson et al. $[\mathbf{3}, 2.12]$ for a detailed proof). We shall only need the easy half of this fact, which is the content of the following:

Proposition 2.11. There exists, for every $N^{\bullet} \in \mathrm{ObC}^{b}(\Lambda$-mod), a functorial quasiisomorphism $N^{\bullet} \rightarrow \mathrm{GF}\left(N^{\bullet}\right)$.

Proof. We consider, firstly, the case of an object $N$ of $\Lambda$-mod. In this case it turns out that $\operatorname{GF}(N)$ is an injective resolution of $N$ in $\Lambda$-mod. Indeed, by definition, $\mathrm{GF}(N)=\operatorname{tot}\left(Y^{\bullet \bullet}\right)$ with $Y^{p, \bullet}=\mathrm{G}\left(\mathrm{F}(N)^{p}\right)=\mathrm{G}\left(S(p) \otimes_{k} N_{p}\right)$, i.e., with

$$
Y^{p q}=S^{p+q}\left(V^{*}\right) \otimes_{k} N_{p} \otimes_{k} \bigwedge\left(V^{*}\right)(q) .
$$

In particular, $\operatorname{GF}(N)^{m}=0$ for $m<0$ and $\operatorname{GF}(N)^{0}=\bigoplus_{p \in \mathbb{Z}} N_{p} \otimes_{k} \bigwedge\left(V^{*}\right)(-p)$.

Let $\beta^{p}: N \rightarrow N_{p} \otimes_{k} \bigwedge\left(V^{*}\right)(-p)$ be the morphism corresponding, according to Lemma 2.2 , to $(-1)^{p} \operatorname{id}_{N_{p}}$, and let $\beta: N \rightarrow \mathrm{GF}(N)^{0}$ be the morphism defined by $\beta^{p}, p \in \mathbb{Z}$. We want to check that $d_{\mathrm{GF}(N)}^{0} \circ \beta=0$. This is equivalent to the fact that, $\forall p \in \mathbb{Z}$, the diagram:

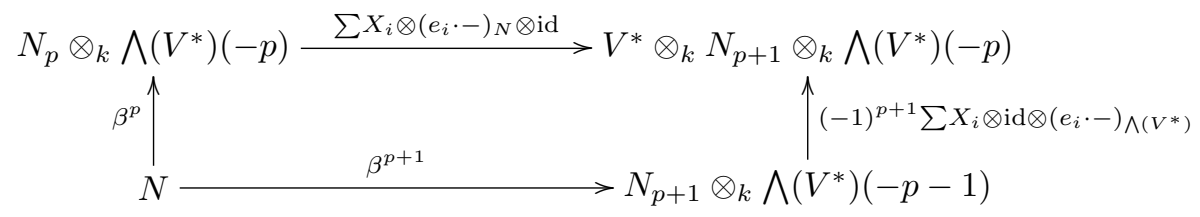


anticommutes. According to Lemma 2.2, this is equivalent to the fact that the diagram:

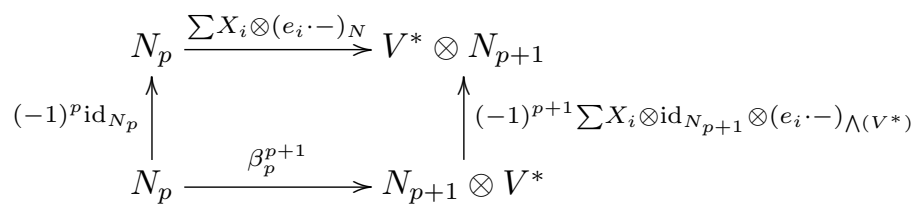

anticommutes. But, according to Cor. 2.3, $\beta_{p}^{p+1}=(-1)^{p+1} \cdot(-1)^{p+1} \sum\left(e_{i} \cdot-\right)_{N} \otimes$ $X_{i}=\sum\left(e_{i} \cdot-\right)_{N} \otimes X_{i}$.

We have thus defined a morphism of complexes $\beta: N \rightarrow \mathrm{GF}(N)$. In order to show that it is a quasi-isomorphism, one may assume, by induction on $\operatorname{dim}_{k} N$, that $N=\underline{k}(a)$ for some $a \in \mathbb{Z}$, and then that $a=0$, i.e., that $N=\underline{k}$. In this case $\mathrm{F}(\underline{k})=S$ and the complex $\mathrm{G}(S)$ :

$$
\cdots \rightarrow 0 \rightarrow \bigwedge\left(V^{*}\right) \rightarrow V^{*} \otimes_{k} \bigwedge\left(V^{*}\right)(1) \rightarrow \cdots \rightarrow S^{p}\left(V^{*}\right) \otimes_{k} \bigwedge\left(V^{*}\right)(p) \rightarrow \cdots
$$

is an injective resolution of $\underline{k}$ in $\Lambda$-mod, as one can easily check using the fact that the Koszul complex

$$
0 \rightarrow S(-n-1) \otimes_{k} \stackrel{n+1}{\wedge} V^{*} \rightarrow \cdots \rightarrow S(-1) \otimes_{k} V^{*} \rightarrow S \rightarrow 0
$$

is a (free) resolution of $S / S_{+}$in $S$-mod.

The general case $N^{\bullet} \in \mathrm{ObC}^{b}(\Lambda$-mod) can be now deduced from the following easy observation: $\operatorname{GF}\left(N^{\bullet}\right)=\operatorname{tot}\left(Z^{\bullet \bullet}\right)$, where $Z^{\bullet \bullet}$ is the double complex with $Z^{p, \bullet}=$ $\mathrm{GF}\left(N^{p}\right)$ and with $d_{Z}^{\prime p}: Z^{p, \bullet} \rightarrow Z^{p+1, \bullet}$ equal to $\mathrm{GF}\left(d_{N}^{p}\right)$. Indeed, by definition, $\mathrm{F}\left(N^{\bullet}\right)=\operatorname{tot}\left(X^{\bullet \bullet}\right)$ where $X^{p, \bullet}=\mathrm{F}\left(N^{p}\right)$ and $\mathrm{GF}\left(N^{\bullet}\right)=\operatorname{tot}\left(Y^{\bullet \bullet}\right)$ where $Y^{m, \bullet}=$ $\mathrm{G}\left(\operatorname{tot}\left(X^{\bullet \bullet}\right)^{m}\right)=\bigoplus_{p+q=m} \mathrm{G}\left(X^{p q}\right)$. Consider the triple complex $W^{\bullet \bullet}$ defined by $W^{p q, \bullet}=\mathrm{G}\left(X^{p q}\right)$. We have that $Z^{p, \bullet}=\mathrm{GF}\left(N^{p}\right)=\mathrm{G}\left(X^{p, \bullet}\right)=\operatorname{tot}\left(W^{p, \bullet \bullet}\right)$, hence $\operatorname{tot}\left(Z^{\bullet \bullet}\right)=\operatorname{tot}\left(W^{\bullet \bullet \bullet}\right)=\operatorname{tot}\left(Y^{\bullet \bullet}\right)=\operatorname{GF}\left(N^{\bullet}\right)$.

Corollary 2.12. $\forall N^{\bullet} \in \mathrm{Ob} \mathrm{C}^{b}(\Lambda$-mod), there exists a functorial quasi-isomorphism:

$$
\mathrm{T}^{-n-1} \mathrm{G}\left(\left(\mathrm{F}\left(N^{\bullet}\right)^{\vee} \otimes_{S} \omega_{S}\right)^{*}\right) \longrightarrow N^{\bullet} \text {. }
$$

Proof. By Proposition 2.11, there exists a quasi-isomorphism $N^{\bullet *} \rightarrow \mathrm{GF}\left(N^{\bullet *}\right)$ and, by Lemma $2.7(\mathrm{~b}), \mathrm{F}\left(N^{\bullet *}\right) \simeq \mathrm{F}\left(N^{\bullet}\right)^{\vee}$. One gets a quasi-isomorphism $\mathrm{G}\left(\mathrm{F}\left(N^{\bullet}\right)^{\vee}\right)^{*} \rightarrow$ $N^{\bullet}$ and, by Lemma $2.7(\mathrm{c}), \mathrm{G}\left(\mathrm{F}\left(N^{\bullet}\right)^{\vee}\right)^{*} \simeq \mathrm{T}^{-n-1} \mathrm{G}\left(\left(\mathrm{F}\left(N^{\bullet}\right)^{\vee} \otimes_{S} \omega_{S}\right)^{*}\right)$.

\section{The Horrocks correspondence}

Definition 3.1. (i) If $M, M^{\prime} \in \mathrm{Ob}\left(S\right.$-mod) let $I_{\mathcal{P}}\left(M^{\prime}, M\right)$ denote the subgroup of $\operatorname{Hom}_{S \text {-mod }}\left(M^{\prime}, M\right)$ consisting of the morphisms factorizing through an object of $\mathcal{P}$. The stable category $S$-mod has, by definition, the same objects as $S$-mod, but the groups Hom are given by:

$$
\underline{\operatorname{Hom}}_{S-\bmod }\left(M^{\prime}, M\right):=\operatorname{Hom}_{S-\bmod }\left(M^{\prime}, M\right) / I_{\mathcal{P}}\left(M^{\prime}, M\right) .
$$

(ii) Similarly, using the full subcategory $\widetilde{\mathcal{P}}$ of Coh $\mathbb{P}$ consisting of finite direct sums of invertible sheaves $\mathcal{O}_{\mathbb{P}}(a), a \in \mathbb{Z}$, one defines the stable category $\underline{\text { Coh }} \mathbb{P}$. 
Definition 3.2. A complex $K^{\bullet} \in \mathrm{ObC}^{b}(\mathcal{P})$ is called a Horrocks complex if it satisfies the following equivalent conditions:

(1) $\mathrm{H}^{i}\left(K^{\bullet}\right)=0$ for $i \leqslant-2$ and $\mathrm{H}^{i}\left(K^{\bullet} \vee\right)=0$ for $i \leqslant 1$

(2) $\mathrm{H}^{i}\left(K^{\bullet \vee}\right)=0$ for $i \leqslant 1$ and $\operatorname{dim} \mathrm{H}^{i}\left(K^{\bullet} \vee\right) \leqslant n+2-i$, for $i>1$

(3) $\mathrm{H}^{i}\left(K^{\bullet}\right)=0$ for $i \leqslant-2$ and $\operatorname{dim} \mathrm{H}^{i}\left(K^{\bullet}\right) \leqslant n-1-i$, for $i \geqslant-1$.

Here "dim" stands for "Krull dimension". The equivalence of these conditions follows from Lemma 3.3 below. Condition (1) implies that if $K^{\prime \bullet} \in \mathrm{ObC}^{b}(\mathcal{P})$ is homotopically equivalent to a Horrocks complex then it is a Horrocks complex.

Let $M \in \mathrm{Ob}\left(S\right.$-mod) and let $L^{\bullet}$ (resp., $\left.L^{\prime \bullet}\right)$ be a free resolution of $M\left(\right.$ resp., $\left.M^{\vee}\right)$ in $S$-mod. One can concatenate the complexes $L^{\prime \bullet}$ and $\mathrm{T}^{-1}\left(L^{\bullet \vee}\right)$ using the composite morphism $L^{\prime 0} \rightarrow M^{\vee} \hookrightarrow L^{0 \vee}$. The dual $K^{\bullet}$ of the resulting complex is a Horrocks complex. We call it a Horrocks resolution of $M$.

Lemma 3.3. Let $A$ be a Noetherian (commutative) ring and let $P^{\bullet}$ be a left bounded complex of finitely generated projective A-modules. Then the following conditions are equivalent:

(i) $\mathrm{H}^{i}\left(P^{\bullet}\right)=0, \forall i<0$

(ii) $\forall i>0, \forall \mathfrak{p} \in \operatorname{Supp}^{i}\left(P^{\bullet \vee}\right) \subseteq \operatorname{Spec} A$, $\operatorname{depth} A_{\mathfrak{p}} \geqslant i$.

Proof. (i) $\Rightarrow$ (ii) Let $i>0$ and let $\mathfrak{p} \in \operatorname{Spec} A$ with $\operatorname{depth} A_{\mathfrak{p}}<i$. Let $M:=C^{0}\left(P^{\bullet}\right):=$ $\operatorname{Coker}\left(P^{-1} \rightarrow P^{0}\right)$. Condition (i) implies that $M$ has finite projective dimension. Now, the Auslander-Buchsbaum formula implies that the projective dimension of the $A_{\mathfrak{p}}$-module $M_{\mathfrak{p}}$ is $\leqslant \operatorname{depth} A_{\mathfrak{p}}<i$, hence $\mathrm{H}^{i}\left(P^{\bullet \vee}\right)_{\mathfrak{p}} \simeq \operatorname{Ext}_{A_{\mathfrak{p}}}^{i}\left(M_{\mathfrak{p}}, A_{\mathfrak{p}}\right)=0$, whence $\mathfrak{p} \notin \operatorname{Supp} \mathrm{H}^{i}\left(P^{\bullet \vee}\right)$.

(ii) $\Rightarrow$ (i) We use induction on $m:=\sup \left\{i \in \mathbb{Z} \mid \mathrm{H}^{i}\left(P^{\bullet \vee}\right) \neq 0\right\}$. The case $m \leqslant 0$ is clear. For the proof of the induction step $(m-1) \rightarrow m$, consider the $A$-module $N:=$ $C^{-1}\left(P^{\bullet}\right):=\operatorname{Coker}\left(P^{-2} \rightarrow P^{-1}\right)$. Applying the induction hypothesis to $\mathrm{T}^{-1} P^{\bullet}$, one gets that $\mathrm{H}^{i}\left(P^{\bullet}\right)=0, \forall i<-1$, hence the sequence:

$$
0 \rightarrow P^{-r} \rightarrow \cdots \rightarrow P^{-2} \rightarrow P^{-1} \rightarrow N \rightarrow 0
$$

is exact. We assert that $\operatorname{Ass}(N) \subseteq \operatorname{Ass}(A)$. Indeed, let $\mathfrak{p} \in \operatorname{Ass}(N)$ and $d:=\operatorname{depth} A_{\mathfrak{p}}$. It follows from the Auslander-Buchsbaum formula that the projective dimension of the $A_{\mathfrak{p}}$-module $N_{\mathfrak{p}}$ is $d$, which implies that $\operatorname{Ext}_{A_{\mathfrak{p}}}^{d}\left(N_{\mathfrak{p}}, A_{\mathfrak{p}}\right) \neq 0$. If $d>0$ then $\operatorname{Ext}_{A_{\mathfrak{p}}}^{d}\left(N_{\mathfrak{p}}, A_{\mathfrak{p}}\right) \simeq \mathrm{H}^{d+1}\left(P^{\bullet \vee}\right)_{\mathfrak{p}}$ hence, by (ii), depth $A_{\mathfrak{p}} \geqslant d+1$, a contradiction. It remains that $d=0$, i.e., $\mathfrak{p} \in \operatorname{Ass}(A)$.

Now, $\mathrm{H}^{-1}\left(P^{\bullet}\right) \simeq \operatorname{Ker}\left(N \rightarrow P^{0}\right)$, hence $\operatorname{Ass}\left(\mathrm{H}^{-1}\left(P^{\bullet}\right)\right) \subseteq \operatorname{Ass}(N) \subseteq \operatorname{Ass}(A)$. If $\mathfrak{p} \in \operatorname{Ass}(A)$ then, by (ii), the sequence:

$$
P_{\mathfrak{p}}^{0 \vee} \rightarrow P_{\mathfrak{p}}^{-1 \vee} \rightarrow \cdots \rightarrow P_{\mathfrak{p}}^{-r \vee} \rightarrow 0
$$

is exact. Since it consists of free $A_{\mathfrak{p}}$-modules, its dual is also exact. In particular, it follows that $\mathrm{H}^{-1}\left(P^{\bullet}\right)_{\mathfrak{p}}=0$. One deduces that $\operatorname{Ass}\left(\mathrm{H}^{-1}\left(P^{\bullet}\right)\right)=\emptyset$, i.e., $\mathrm{H}^{-1}\left(P^{\bullet}\right)=0$.

Theorem 3.4. The functor $C^{-1}: \mathrm{C}^{b}(\mathcal{P}) \rightarrow S$-mod associating to a complex $L^{\bullet}$ the cokernel of the differential $d_{L}^{-2}: L^{-2} \rightarrow L^{-1}$ induces a functor $\underline{C}^{-1}: \mathrm{K}^{b}(\mathcal{P}) \rightarrow S$-mod which, restricted to the full subcategory $\mathcal{H}$ of $\mathrm{K}^{b}(\mathcal{P})$ consisting of Horrocks complexes, is an equivalence of categories. 
Proof. If a morphism $f: L^{\prime \bullet} \rightarrow L^{\bullet}$ in $\mathrm{C}^{b}(\mathcal{P})$ is homotopic to 0 then $C^{-1}\left(L^{\prime \bullet}\right) \rightarrow$ $C^{-1}\left(L^{\bullet}\right)$ factorizes through $L^{-1} \rightarrow C^{-1}\left(L^{\bullet}\right)$ (and through $C^{-1}\left(L^{\prime \bullet}\right) \rightarrow L^{\prime 0}$ ) hence $C^{-1}$ induces a functor $C^{-1}$ as in the statement.

The fact that $\underline{C}^{-1} \mid \overline{\mathcal{H}}$ is fully faithful follows from the more general Lemma 3.5 below. The fact that $\underline{C^{-1}} \mid \mathcal{H} \rightarrow S$-mod is essentially surjective was already observed in the last part of Definition 3.2.

Lemma 3.5. Let $L^{\bullet}, L^{\prime \bullet} \in \mathrm{ObC}(\mathcal{P})$. If $\mathrm{H}^{i}\left(L^{\bullet}\right)=0$ for $i \leqslant-2$ and if $\mathrm{H}^{i}\left(L^{\prime \bullet \vee}\right)=0$ for $i \leqslant 1$, then the morphism:

$$
\operatorname{Hom}_{\mathrm{C}(\mathcal{P})}\left(L^{\prime \bullet}, L^{\bullet}\right) \longrightarrow \operatorname{Hom}_{S-\bmod }\left(C^{-1}\left(L^{\prime \bullet}\right), C^{-1}\left(L^{\bullet}\right)\right)
$$

is surjective and induces an isomorphism:

$$
\operatorname{Hom}_{\mathrm{K}(\mathcal{P})}\left(L^{\prime \bullet}, L^{\bullet}\right) \stackrel{\sim}{\longrightarrow} \underline{\operatorname{Hom}}_{S-\bmod }\left(C^{-1}\left(L^{\prime \bullet}\right), C^{-1}\left(L^{\bullet}\right)\right) .
$$

Proof. The complex $\cdots \rightarrow L^{-2} \rightarrow L^{-1} \rightarrow 0$ is a free resolution of $C^{-1}\left(L^{\bullet}\right)$ in $S$-mod, and the complex $\cdots \rightarrow L^{\prime 1 \vee} \rightarrow L^{\prime 0 \vee} \rightarrow 0$ is a free resolution of $C^{-1}\left(L^{\prime \bullet}\right)^{\vee}$. Now, one can use the following two elementary facts: (1) if $P^{\bullet} \in \mathrm{ObC}^{\leqslant 0}(\mathcal{P})$, $M^{\bullet} \in \mathrm{ObC}^{\leqslant 0}\left(S\right.$-mod) and $\mathrm{H}^{i}\left(M^{\bullet}\right)=0$ for all $i<0$, then any morphism $C^{0}\left(P^{\bullet}\right) \rightarrow$ $C^{0}\left(M^{\bullet}\right)$ can be lifted to a morphism of complexes $P^{\bullet} \rightarrow M^{\bullet} ;$ (2) if, moreover, $C^{0}\left(P^{\bullet}\right) \rightarrow C^{0}\left(M^{\bullet}\right)$ factorizes through an object of $\mathcal{P}$ (hence through $M^{0} \rightarrow C^{0}\left(M^{\bullet}\right)$ ) then the morphism of augmented complexes:

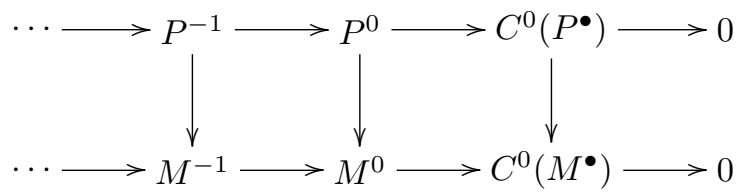

is homotopic to 0 .

Theorem 3.6. For $N^{\bullet} \in \mathrm{Ob} \mathrm{C}^{b}\left(\Lambda\right.$-mod) the complex $\mathrm{F}\left(N^{\bullet}\right)$ is a Horrocks complex if and only if the linear part of a minimal free resolution of $N^{\bullet}$ in $\Lambda$-mod is of the form $\bigoplus_{i=-1}^{n-1} \mathrm{~T}^{-i} \mathrm{G}\left(H^{i}\right)$, where $H^{i}$ is the $k$-vector space graded dual of a finitely generated graded $S$-module of Krull dimension $\leqslant i+1, i=-1, \ldots, n-1$.

Proof. Let us denote $\mathrm{F}\left(N^{\bullet}\right)$ by $K^{\bullet}$. By Corollary 2.12 and by Lemma 2.9(b), the linear part of a minimal free resolution of $N^{\bullet}$ is isomorphic to $\bigoplus_{i \in \mathbb{Z}} \mathrm{T}^{-i} \mathrm{G}\left(H^{i}\right)$, where $H^{i}=\mathrm{H}^{i}\left(\mathrm{~T}^{-n-1}\left(\left(K^{\bullet \vee} \otimes_{S} \omega_{S}\right)^{*}\right)\right) \simeq\left(\mathrm{H}^{n+1-i}\left(K^{\bullet \vee} \otimes_{S} \omega_{S}\right)\right)^{*}$. One can now conclude, using condition (2) from Definition 3.2.

Definition 3.7. A minimal complex $G^{\bullet} \in \mathrm{ObC}^{-}(\mathcal{I})$ with $\mathrm{H}^{p}\left(G^{\bullet}\right)=0$ for $p \ll 0$ is called a Horrocks-Trautmann complex if it satisfies the following conditions (compare with $[\mathbf{9}, 1.6])$ :

(1) $F_{n-1} G^{\bullet}=G^{\bullet}$ and $F_{0} G^{\bullet}=0$, i.e., $G^{p} \simeq \bigoplus_{i=1}^{n-1} \bigwedge\left(V^{*}\right)(p-i)^{c_{p i}}, \forall p \in \mathbb{Z}$,

(2) $\lim _{p \rightarrow \infty}\left(c_{-p, i} / p^{i+1}\right)=0, i=1, \ldots, n-1$.

Lemma 3.8. A minimal complex $G^{\bullet} \in \mathrm{ObC}^{-}(\mathcal{I})$ is a Horrocks-Trautmann complex if and only if its linear part is of the form $\bigoplus_{i=1}^{n-1} \mathrm{~T}^{-i} \mathrm{G}\left(H^{i}\right)$, where $H^{i}$ is the k-vector space graded dual of a finitely generated graded $S$-module of Krull dimension $\leqslant i+1$, $i=1, \ldots, n-1$. 
Proof. The equivalence can be proved by applying to $G^{\bullet *}$ the following:

Assertion. For a minimal complex $I^{\bullet} \in \mathrm{ObC}^{+}(\mathcal{I})$, the following conditions are equivalent:

(i) $\mathrm{H}^{p}\left(I^{\bullet}\right)=0$, for $p \gg 0$

(ii) The linear part of $I^{\bullet}$ is of the form $\bigoplus_{i \in \mathbb{Z}} \mathrm{T}^{-i} \mathrm{G}\left(M^{i}\right)$, where $M^{i}$ is a finitely generated graded $S$-module, $\forall i \in \mathbb{Z}$.

(i) $\Rightarrow\left(\right.$ ii) $\mathrm{H}^{p}\left(I^{\bullet}\right)=0, \forall p>m$, for some $m \in \mathbb{Z}$. Let $Z^{m}:=\operatorname{Ker}\left(I^{m} \rightarrow I^{m+1}\right) . \sigma^{\geqslant m} I^{\bullet}$ is a minimal right free resolution of $\mathrm{T}^{-m} Z^{m}$ in $\Lambda$-mod. By Proposition 2.11, the complex $\mathrm{GF}\left(\mathrm{T}^{-m} Z^{m}\right)$ is a right free resolution of $\mathrm{T}^{-m} Z^{m}$ in $\Lambda$-mod. From Lemma 2.9(b), it can be contracted to a minimal complex $J^{\bullet}$ in $\mathrm{C}^{+}(\mathcal{I})$, whose linear part is

$$
\bigoplus_{i \in \mathbb{Z}} \mathrm{T}^{-i} \mathrm{G}\left(\mathrm{H}^{i}\left(\mathrm{~F}\left(\mathrm{~T}^{-m} Z^{m}\right)\right)\right)=\bigoplus_{i \in \mathbb{Z}} \mathrm{T}^{-i} \mathrm{G}\left(\mathrm{H}^{i-m}\left(\mathrm{~F}\left(Z^{m}\right)\right)\right) .
$$

$\sigma^{\geqslant m} I^{\bullet}$ and $J^{\bullet}$ are isomorphic in $\mathrm{D}^{+}(\Lambda$-mod $)$ hence, since every free object of $\Lambda$-mod is an injective object of this category, they are isomorphic in $\mathrm{K}^{+}(\mathcal{I})$ and consequently, by 2.8(iii), isomorphic in $\mathrm{C}^{+}(\mathcal{I})$. One deduces that the linear part of $\sigma^{\geqslant m} I^{\bullet}$ is isomorphic to $\bigoplus_{i \in \mathbb{Z}} \mathrm{T}^{-i} \mathrm{G}\left(\mathrm{H}^{i-m}\left(\mathrm{~F}\left(Z^{m}\right)\right)\right)$.

(ii) $\Rightarrow$ (i) It suffices to prove that if $M \in \mathrm{Ob}\left(S\right.$-mod) then $\mathrm{H}^{p}(\mathrm{G}(M))=0$ for $p \gg 0$. Let $L^{\bullet}$ be a finite free resolution of $M$ in $S$-mod. $\mathrm{G}(M)$ and $\mathrm{G}\left(L^{\bullet}\right)$ are quasiisomorphic (even homotopically equivalent). Since $\mathrm{G}(S)$ is a right free resolution of $\underline{k}$ in $\Lambda$-mod (see the proof of Proposition 2.11) it follows that $\mathrm{H}^{p}\left(\mathrm{G}\left(L^{\bullet}\right)\right)=0$ for $p \gg 0$.

Theorem 3.9. There exists an equivalence of categories between the full subcategory $\mathcal{H} \mathcal{T}$ of $\mathrm{K}^{-}(\mathcal{I})$ consisting of Horrocks-Trautmann complexes and the full subcategory of $\underline{\mathrm{Coh}} \mathbb{P}(V)$ consisting of the coherent sheaves $\mathcal{F}$ with the property that $\mathrm{H}^{0}(\mathcal{F}(-t))=0$, for $t \gg 0$.

Proof. The equivalence from the statement will appear as a composition of previously established equivalences.

(1) Let $\mathcal{B}$ be the full subcategory of $\operatorname{Coh} \mathbb{P}(V)$ consisting of the coherent sheaves with the property from the statement. Let $\mathcal{A}$ be the full subcategory of $S$-mod consisting of the modules of projective dimension $\leqslant n-1$. Using Theorem 1.1 (Graded Serre Duality) one sees that the functor $(-)^{\sim}: S$-mod $\rightarrow \operatorname{Coh} \mathbb{P}(V)$ induces an equivalences of categories between $\mathcal{A}$ and $\mathcal{B}$. Moreover, this equivalence induces an equivalence between the correponding full subcategories $\underline{\mathcal{A}}$ and $\underline{\mathcal{B}}$ of $S$ - $\underline{\bmod }$ and $\underline{\operatorname{Coh}} \mathbb{P}(V)$, respectively.

(2) By Lemma 1.2, the equivalence $\underline{C}^{-1}: \mathcal{H} \rightarrow S$-mod from Theorem 3.4 induces an equivalence between the full subcategory $\mathcal{H}^{\prime}$ of $\mathcal{H}$ consisting of the Horrocks complexes $K^{\bullet}$ with the additional property that $\mathrm{H}^{i}\left(K^{\bullet} \vee\right)=0$ for $i \geqslant n+1$ and $\underline{\mathcal{A}}$.

(3) Finally, there is a well-known equivalence $\Phi$ between the full subcategory $\mathcal{K}$ of $\mathrm{K}^{-}(\mathcal{I})$ consisting of the complexes $I^{\bullet}$ with $\mathrm{H}^{p}\left(I^{\bullet}\right)=0$ for $p \ll 0$ and $\mathrm{D}^{b}(\Lambda$-mod $)$. $\Phi$ associates to $I^{\bullet}$ a convenient truncation $\tau^{\geqslant m} I^{\bullet}$ with $m \ll 0$ (it suffices that $\mathrm{H}^{p}\left(I^{\bullet}\right)=$ 0 for $p<m$ ) and its quasi-inverse associates to a complex in $\mathrm{D}^{b}(\Lambda$-mod) a free resolution of it. Now, by Theorem 3.6 and Lemma 3.8, the composition of the BGG equivalence (Theorem 2.10) $\mathrm{F}: \mathrm{D}^{b}(\Lambda-\bmod ) \rightarrow \mathrm{K}^{b}(\mathcal{P})$ and $\Phi$ induces an equivalence between $\mathcal{H} \mathcal{T}$ and $\mathcal{H}^{\prime}$. 
Example 3.10. (Eilenberg-MacLane sheaves)

Let $0<i<n$ and let $E$ be a finitely generated graded $S$-module of Krull dimension $\leqslant i+1$. Consider a minimal free resolution of $E$ in $S$-mod:

$$
0 \rightarrow Q^{-n-1} \rightarrow \cdots \rightarrow Q^{0} \rightarrow E \rightarrow 0 .
$$

Applying Lemma 3.3 to $P^{\bullet}:=\mathrm{T}^{n-i}\left(Q^{\bullet \vee}\right)$ one derives that $\mathrm{H}^{j}\left(Q^{\bullet \vee}\right)=0$ for $j \leqslant n-i-1$. Using condition (1) from Definition 3.2 one deduces that $K^{\bullet}:=\mathrm{T}^{n-i+1}\left(Q^{\bullet}\right)$ is a Horrocks complex. It is a Horrocks resolution of $M:=\operatorname{Coker}\left(\left(Q^{-n+i+1}\right)^{\vee} \rightarrow\left(Q^{-n+i}\right)^{\vee}\right)$. $M$ has a minimal free resolution:

$$
0 \rightarrow\left(Q^{0}\right)^{\vee} \rightarrow \cdots \rightarrow\left(Q^{-n+i+1}\right)^{\vee} \rightarrow\left(Q^{-n+i}\right)^{\vee} \rightarrow M \rightarrow 0 .
$$

Let $\mathcal{F}:=\widetilde{M}$. Since $\mathrm{H}^{j}\left(K^{\bullet \vee}\right)=0$ for $j \neq n-i+1$ and $\mathrm{H}^{n-i+1}\left(K^{\bullet \vee}\right) \simeq E$, it follows from the proof of Theorem 3.6 that the Horrocks-Trautmann complex associated to $\mathcal{F}$ is $\mathrm{T}^{-i} \mathrm{G}(H)$ where $H=\left(E \otimes_{S} \omega_{S}\right)^{*}$. Moreover, by Graded Serre Duality (Theorem 1.1), $\mathrm{H}_{*}^{j} \mathcal{F}=0$ for $0<j<n, j \neq i$, and $\mathrm{H}_{*}^{i} \mathcal{F} \simeq H$.

When $E$ is of finite length $\mathcal{F}$ is a locally free sheaf. The locally free sheaves of this kind were called Eilenberg-MacLane bundles in Horrocks [16].

\section{The Horrocks correspondence and the BGG correspondence}

Definition 4.1. The geometric $B G G$ functor is the functor $\mathrm{L}: \Lambda$-mod $\rightarrow \mathrm{C}^{b}(\mathrm{Coh} \mathbb{P})$ defined by $\mathrm{L}(N):=\mathrm{F}(N)^{\sim}$.

We denote by $\Lambda$-mod the stable category of $\Lambda$-mod with respect to its full subcategory $\mathcal{I}$ consisting of free objects (see Definition 3.1).

Lemma 4.2. If $N, N^{\prime} \in \mathrm{Ob}(\Lambda$-mod $)$ then, $\forall p \geqslant 1$ :

$$
\operatorname{Hom}_{\mathrm{K}^{b}(\mathbb{P})}\left(\mathrm{L}\left(N^{\prime}\right), \mathrm{T}^{p} \mathrm{~L}(N)\right) \stackrel{\sim}{\longrightarrow} \operatorname{Hom}_{\mathrm{D}^{b}(\mathbb{P})}\left(\mathrm{L}\left(N^{\prime}\right), \mathrm{T}^{p} \mathrm{~L}(N)\right) .
$$

Proof. The lemma is an immediate application of Lemma B.4, taking into account that $\mathrm{H}^{i}\left(\mathcal{O}_{\mathbb{P}}(a)\right)=0$ for $i>0, i \neq n, \forall a \in \mathbb{Z}$, and $\mathrm{H}^{n}\left(\mathcal{O}_{\mathbb{P}}(a)\right)=0, \forall a \geqslant-n$.

Corollary 4.3. If $N, N^{\prime} \in \mathrm{Ob}(\Lambda$-mod $)$ then, $\forall p \geqslant 1$ :

$$
\operatorname{Hom}_{\mathrm{D}^{b}(\Lambda)}\left(N^{\prime}, \mathrm{T}^{p} N\right) \stackrel{\sim}{\longrightarrow} \operatorname{Hom}_{\mathrm{D}^{b}(\mathbb{P})}\left(\mathrm{L}\left(N^{\prime}\right), \mathrm{T}^{p} \mathrm{~L}(N)\right) .
$$

Proof. By the BGG correspondence for graded modules Theorem 2.10:

$$
\operatorname{Hom}_{\mathrm{D}^{b}(\Lambda)}\left(N^{\prime}, \mathrm{T}^{p} N\right) \stackrel{\sim}{\longrightarrow} \operatorname{Hom}_{\mathrm{K}^{b}(\mathcal{P})}\left(\mathrm{F}\left(N^{\prime}\right), \mathrm{T}^{p} \mathrm{~F}(N)\right)
$$

and, on the other hand, it is obvious that:

$$
\operatorname{Hom}_{\mathrm{K}^{b}(\mathcal{P})}\left(\mathrm{F}\left(N^{\prime}\right), \mathrm{T}^{p} \mathrm{~F}(N)\right) \stackrel{\sim}{\longrightarrow} \operatorname{Hom}_{\mathrm{K}^{b}(\mathbb{P})}\left(\mathrm{L}\left(N^{\prime}\right), \mathrm{T}^{p} \mathrm{~L}(N)\right) .
$$

It only remains, now, to apply Lemma 4.2.

The following theorem is the Bernstein-Gel'fand-Gel'fand correspondence for coherent sheaves on projective spaces. We include here a direct proof of this result.

Theorem 4.4 ([4, Theorem 2]). The functor $\mathrm{L}: \Lambda-\bmod \rightarrow \mathrm{C}^{b}(\mathrm{Coh} \mathbb{P})$ induces an equivalence of categories $\mathrm{L}: \Lambda$ - $\underline{\mathrm{mod}} \rightarrow \mathrm{D}^{b}(\mathrm{Coh} \mathbb{P})$. 
Proof. $\mathrm{L}\left(\bigwedge\left(V^{*}\right)\right)$ is the tautological Koszul complex on $\mathbb{P}(V)$ :

$$
0 \rightarrow \mathcal{O}_{\mathbb{P}}(-n-1) \otimes_{k} \stackrel{n+1}{\wedge} V^{*} \rightarrow \cdots \rightarrow \mathcal{O}_{\mathbb{P}}(-1) \otimes_{k} V^{*} \rightarrow \mathcal{O}_{\mathbb{P}} \rightarrow 0
$$

hence, if $P$ is a free object of $\Lambda$-mod then $\mathrm{L}(P)$ is an acyclic complex. It follows that $\mathrm{L}: \Lambda$-mod $\rightarrow \mathrm{C}^{b}(\operatorname{Coh} \mathbb{P})$ induces a functor $\mathrm{L}: \Lambda$ - $\underline{\bmod } \rightarrow \mathrm{D}^{b}(\operatorname{Coh} \mathbb{P})$.

We firstly show that the induced functor is fully faithful. Let $N, N^{\prime} \in \operatorname{Ob}(\Lambda$-mod). We have to show that the morphism:

$$
\operatorname{Hom}_{\Lambda-\bmod }\left(N^{\prime}, N\right) \longrightarrow \operatorname{Hom}_{\mathrm{D}^{b}(\mathbb{P})}\left(\mathrm{L}\left(N^{\prime}\right), \mathrm{L}(N)\right)
$$

is surjective and that its kernel consists of the morphisms factorizing through a free object of $\Lambda$-mod. Consider an exact sequence $0 \rightarrow K \rightarrow P \rightarrow N \rightarrow 0$ with $P$ a free object of $\Lambda$-mod. From Lemma 4.2:

$$
\operatorname{Hom}_{\mathrm{K}^{b}(\mathbb{P})}\left(\mathrm{L}\left(N^{\prime}\right), \mathrm{TL}(P)\right) \stackrel{\sim}{\longrightarrow} \operatorname{Hom}_{\mathrm{D}^{b}(\mathbb{P})}\left(\mathrm{L}\left(N^{\prime}\right), \mathrm{TL}(P)\right)
$$

and $\operatorname{Hom}_{\mathrm{D}^{b}(\mathbb{P})}\left(\mathrm{L}\left(N^{\prime}\right), \mathrm{TL}(P)\right)=0$ since $\mathrm{L}(P)$ is acyclic. Now, applying the functors $\operatorname{Hom}_{\mathrm{K}^{b}(\mathbb{P})}\left(\mathrm{L}\left(N^{\prime}\right),-\right)$ and $\operatorname{Hom}_{\mathrm{D}^{b}(\mathbb{P})}\left(\mathrm{L}\left(N^{\prime}\right),-\right)$ to the complex in $\mathrm{K}^{b}(\mathbb{P})$ :

$$
\mathrm{L}(P) \rightarrow \mathrm{L}(N) \rightarrow \mathrm{TL}(K) \rightarrow \mathrm{TL}(P)
$$

deduced (see [8, 2(i),(ii)]) from the semi-split short exact sequence:

$$
0 \rightarrow \mathrm{L}(K) \rightarrow \mathrm{L}(P) \rightarrow \mathrm{L}(N) \rightarrow 0,
$$

one gets a commutative diagram with exact rows:

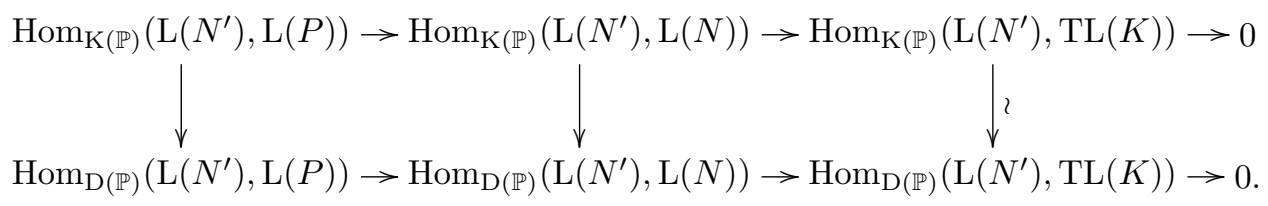

By Lemma 4.2, the vertical arrow from the right hand side of the diagram is an isomorphism. Moreover, $\operatorname{Hom}_{\mathrm{D}(\mathbb{P})}\left(\mathrm{L}\left(N^{\prime}\right), \mathrm{L}(P)\right)=0$ because $\mathrm{L}(P)$ is acyclic. Since the vertical arrows in the commutative diagram:

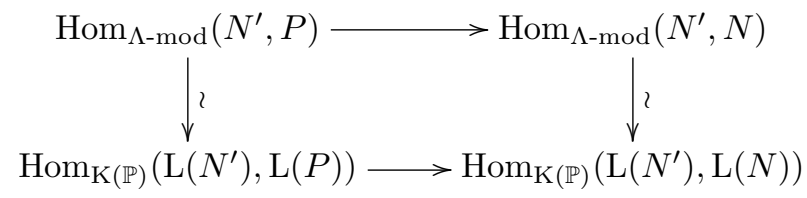

are clearly isomorphisms, one deduces that the morphism $\left(^{*}\right)$ is surjective and that its kernel consists of the morphisms factorizing through $P \rightarrow N$.

The essential surjectivity of $\mathrm{L}: \Lambda$ - $\bmod \rightarrow \mathrm{D}^{b}(\mathbb{P})$ can now be checked, in a wellknown manner, using the following observations:

(1) By what has been proven, the image of $\mathrm{L}$ is a full subcategory of $\mathrm{D}^{b}(\mathbb{P})$.

(2) If $N \in \mathrm{Ob}(\Lambda$-mod) and one considers a short exact sequence $0 \rightarrow N$ $\rightarrow I \rightarrow Q \rightarrow 0$ with $I$ a free object of $\Lambda$-mod then the connecting morphism 
$w: \mathrm{L}(Q) \rightarrow \mathrm{TL}(N)$ deduced (see $[\mathbf{8},(2)(\mathrm{ii})])$ from the semi-split short exact sequence:

$$
0 \rightarrow \mathrm{L}(N) \rightarrow \mathrm{L}(I) \rightarrow \mathrm{L}(Q) \rightarrow 0
$$

is a quasi-isomorphism because $\mathrm{L}(I)$ is acyclic. Similarly, considering a short exact sequence $0 \rightarrow K \rightarrow P \rightarrow N \rightarrow 0$ with $P$ a free object of $\Lambda$-mod one gets a quasiisomorphism $\mathrm{T}^{-1} \mathrm{~L}(N) \rightarrow \mathrm{L}(K)$.

(3) Let $u: N^{\prime} \rightarrow N$ be a morphism in $\Lambda$-mod. Consider an embedding $v: N^{\prime} \rightarrow I^{\prime}$ of $N^{\prime}$ into a free object $I^{\prime}$ of $\Lambda$-mod and define $C \in \mathrm{Ob}(\Lambda$-mod) by the short exact sequence:

$$
0 \rightarrow N^{\prime} \stackrel{(u, v)}{\longrightarrow} N \oplus I^{\prime} \longrightarrow C \rightarrow 0 .
$$

By applying $L$ to this short exact sequence one gets a semi-split short exact sequence, hence $\mathrm{L}(C)$ is homotopically equivalent to $\operatorname{Con} \mathrm{L}((u, v))$. Moreover, $\operatorname{Con} \mathrm{L}((u, v))$ is quasi-isomorphic to $\operatorname{Con} \mathrm{L}(u)$ because $\mathrm{L}\left(I^{\prime}\right)$ is acyclic, whence one gets a quasiisomorphism $\mathrm{L}(C) \rightarrow \operatorname{Con} \mathrm{L}(u)$.

(4) $\mathrm{L}(\underline{k}(-a))=\mathrm{T}^{-a} \mathcal{O}_{\mathbb{P}}(a), \forall a \in \mathbb{Z}$.

Using these observations and the fact that every coherent sheaf on $\mathbb{P}(V)$ has a finite resolution with finite direct sums of invertible sheaves $\mathcal{O}_{\mathbb{P}}(a)$, one deduces immediately (as, for example, in the last part of the proof of $[\mathbf{8}$, Theorem 7]) that $\mathrm{L}: \Lambda-\underline{\bmod } \rightarrow \mathrm{D}^{b}(\mathbb{P})$ is essentially surjective.

Corollary 4.5 ([4, Remark 3 after Theorem 1]). If $\mathcal{F}^{\bullet} \in \mathrm{Ob} \mathrm{C}^{b}(\mathrm{Coh} \mathbb{P})$ then there exists $N \in \mathrm{Ob}\left(\Lambda\right.$-mod) annihilated by $\operatorname{soc}(\Lambda)={ }^{n+1} V$ such that $\mathcal{F}^{\bullet} \simeq \mathrm{L}(N)$ in the derived category $\mathrm{D}^{b}(\mathrm{Coh} \mathbb{P})$. Moreover, $N$ is unique up to isomorphism.

For a proof see, for example, $[\mathbf{8}, 8]$.

Corollary 4.6. If $\mathcal{F}^{\bullet}$ and $N$ are as in Corollary 4.5 then, $\forall i \in \mathbb{Z}, \mathrm{H}^{i}(\mathrm{~F}(N)) \simeq$ $\bigoplus_{j \geqslant-i} \mathbb{H}^{i}\left(\mathcal{F}^{\bullet}(j)\right)$ as $S$-modules (where $\mathbb{H}$ denotes the hypercohomology).

Proof. $\mathrm{H}^{i}(\mathrm{~F}(N))_{j}=0$ for $j<-i$ because $\mathrm{F}(N)^{i}=S(i) \otimes_{k} N_{i}$. For every $j$ one has:

$$
\mathrm{H}^{i}(\mathrm{~F}(N))_{j} \simeq \operatorname{Hom}_{\mathrm{K}^{b}(\mathbb{P})}\left(\mathrm{T}^{-i} \mathcal{O}_{\mathbb{P}}, \mathrm{L}(N)(j)\right)
$$

hence it remains to show that for $j \geqslant-i$ :

$$
\operatorname{Hom}_{\mathrm{K}^{b}(\mathbb{P})}\left(\mathrm{T}^{-i} \mathcal{O}_{\mathbb{P}}, \mathrm{L}(N)(j)\right) \stackrel{\sim}{\longrightarrow} \operatorname{Hom}_{D^{b}(\mathbb{P})}\left(\mathrm{T}^{-i} \mathcal{O}_{\mathbb{P}}, \mathrm{L}(N)(j)\right)
$$

or, equivalently, that:

$$
\operatorname{Hom}_{\mathrm{K}^{b}(\mathbb{P})}\left(\mathrm{L}(\underline{k}(-i)), \mathrm{T}^{i+j} \mathrm{~L}(N(-i-j))\right) \stackrel{\sim}{\longrightarrow} \operatorname{Hom}_{\mathrm{D}^{b}(\mathbb{P})}\left(\mathrm{L}(\underline{k}(-i)), \mathrm{T}^{i+j} \mathrm{~L}(N(-i-j))\right) .
$$

For $j>-i$ this follows from Lemma 4.2. For $j=-i$, the above morphism can be identified with the morphism:

$$
\operatorname{Hom}_{\Lambda-\bmod }(\underline{k}(-i), N) \longrightarrow \operatorname{Hom}_{\mathrm{D}^{b}(\mathbb{P})}(\mathrm{L}(\underline{k}(-i)), \mathrm{L}(N)) .
$$

By Theorem 4.4, the last morphism is surjective and its kernel consists of the composite morphisms $\underline{k}(-i) \rightarrow P \rightarrow N$ with $P$ a free object of $\Lambda$-mod. But the image of $\underline{k}(-i) \rightarrow P$ must lie in $\operatorname{soc}(P)=\operatorname{soc}(\Lambda) \cdot P$. Since $N$ is annihilated by $\operatorname{soc}(\Lambda)$, any such composite morphism must be 0 . 
Definition 4.7. Let $N$ be an object of $\Lambda$-mod annihilated by $\operatorname{soc}(\Lambda)$. Let $P^{\bullet}$ (resp., $P^{\prime \bullet}$ ) be a minimal free resolution of $N$ (resp., $N^{*}$ ) in $\Lambda$-mod. By concatenating the complexes $P^{\prime \bullet}$ and $\mathrm{T}^{-1}\left(P^{\bullet *}\right)$ using the composite morphism $P^{\prime 0} \rightarrow N^{*} \hookrightarrow P^{0 *}$ one gets an acyclic complex which is minimal (since $N^{*}$ is annihilated by $\operatorname{soc}(\Lambda)$, the image of the above composite morphism is contained in $\left.\Lambda_{+} \cdot P^{0 *}\right)$. The $k$-vector space dual $I^{\bullet}$ of this complex (see Definition 2.5) is called a Tate resolution of $N$.

The next theorem, which is one of the main results of the paper of Eisenbud, Fløystad and Schreyer [12], is a direct consequence of Corollary 4.6.

Theorem 4.8 ([12, Theorem 4.1]). If $\mathcal{F}^{\bullet}$ and $N$ are as in Corollary 4.5 and if $I^{\bullet}$ is a Tate resolution of $N$ then the linear part of $I^{\bullet}$ is isomorphic to $\bigoplus_{i \in \mathbb{Z}} \mathrm{T}^{-i} \mathrm{G}\left(\mathbb{H}_{*}^{i} \mathcal{F} \bullet\right)$.

Proof. Let $Z^{-m}:=\operatorname{Ker}\left(I^{-m} \rightarrow I^{-m+1}\right), m>0$. As we have shown in the proof of Lemma 3.8, the linear part of $\sigma^{\geqslant-m} I^{\bullet}$ is isomorphic to $\bigoplus_{i \in \mathbb{Z}} \mathrm{T}^{-i} \mathrm{G}\left(\mathrm{H}^{i+m}\left(\mathrm{~F}\left(Z^{-m}\right)\right)\right)$.

Now, by definition, $N \simeq \operatorname{Coker}\left(I^{-2} \rightarrow I^{-1}\right)$ and since $I^{\bullet}$ is acyclic, $N \simeq$ $\operatorname{Ker}\left(I^{0} \rightarrow I^{1}\right)$. It follows, from observation (2) in the second part of the proof of Theorem 4.4, that $\mathrm{L}\left(Z^{-m}\right) \simeq \mathrm{T}^{-m} \mathrm{~L}(N) \simeq \mathrm{T}^{-m} \mathcal{F}^{\bullet}$ in $\mathrm{D}^{b}(\mathbb{P})$. Moreover, since $Z^{-m}$ is contained in $\Lambda_{+} \cdot I^{-m}$, it is annihilated by $\operatorname{soc}(\Lambda)$. Corollary 4.6 now implies that $\mathrm{H}^{i+m}\left(\mathrm{~F}\left(Z^{-m}\right)\right) \simeq \bigoplus_{j \geqslant-i-m} \mathbb{H}^{i+m}\left(\left(\mathrm{~T}^{-m} \mathcal{F}^{\bullet}\right)(j)\right)=\bigoplus_{j \geqslant-i-m} \mathbb{H}^{i}\left(\mathcal{F}^{\bullet}(j)\right)$. Taking into account what has been recalled in the first paragraph, one deduces that the linear part of $\sigma^{\geqslant-m} I^{\bullet}$ is isomorphic to $\bigoplus_{i \in \mathbb{Z}} \mathrm{T}^{-i} \mathrm{G}\left(\bigoplus_{j \geqslant-i-m} \mathbb{H}^{i}\left(\mathcal{F}^{\bullet}(j)\right)\right)$. Finally, letting $m \rightarrow \infty$ one gets the desired conclusion.

Theorem 4.9. Let $\mathcal{F}$ be a coherent sheaf on $\mathbb{P}^{n}$ with $\mathrm{H}^{0} \mathcal{F}(-t)=0$ for $t \gg 0$, let $M:=\mathrm{H}_{*}^{0} \mathcal{F}$ and let $0 \rightarrow L^{-n+1} \rightarrow \cdots \rightarrow L^{0} \rightarrow M \rightarrow 0$ be a minimal free resolution of $M$ in $S$-mod. Let $N \in \mathrm{Ob}\left(\Lambda\right.$-mod) be as in Corollary 4.5 and let $I^{\bullet}$ be a Tate resolution of $N$. Then:

(a) $I^{\bullet} / F_{0} I^{\bullet}$ is a contraction of $\mathrm{T}^{-n} \mathrm{G}\left(\left(L^{\bullet \vee} \otimes_{S} \omega_{S}\right)^{*}\right)$.

(b) The Horrocks-Trautmann complex corresponding to $\mathcal{F}$ via the equivalence of categories from Theorem 3.9 is isomorphic to $F_{n-1} I^{\bullet} / F_{0} I^{\bullet}$.

Proof. (a) Choose $m \in \mathbb{Z}$ such that $\mathrm{H}^{i}(\mathcal{F}(j))=0, \forall i>0, \forall j \geqslant m-i$. Since, as a consequence of Theorem 4.8, $I^{p} \simeq \bigoplus_{i=0}^{n} \mathrm{H}^{i}(\mathcal{F}(p-i)) \otimes_{k} \bigwedge\left(V^{*}\right)(p-i), \forall p \in \mathbb{Z}$, one sees that $I^{p}=F_{0} I^{p}$ for $p \geqslant m$, hence $I^{\bullet} / F_{0} I^{\bullet}=\left(\sigma^{<m} I^{\bullet}\right) / F_{0}\left(\sigma^{<m} I^{\bullet}\right)$.

Now, let $Z^{m}:=\operatorname{Ker}\left(I^{m} \rightarrow I^{m+1}\right)$. $\mathrm{T}^{-1}\left(\sigma^{<m} I^{\bullet}\right)$ is a minimal (left) free resolution of $\mathrm{T}^{-m} Z^{m}$. One deduces, from Corollary 2.12 , that $\mathrm{T}^{-1}\left(\sigma^{<m} I^{\bullet}\right)$ is a contraction of $\mathrm{T}^{-n-1} \mathrm{G}\left(\left(\mathrm{F}\left(\mathrm{T}^{-m} Z^{m}\right)^{\vee} \otimes_{S} \omega_{S}\right)^{*}\right)$. It follows that $\sigma^{<m} I^{\bullet}$ is a contraction of the complex $\mathrm{T}^{-n} \mathrm{G}\left(\left(\mathrm{F}\left(\mathrm{T}^{-m} Z^{m}\right)^{\vee} \otimes_{S} \omega_{S}\right)^{*}\right)$.

Let $F^{\bullet}:=\mathrm{F}\left(\mathrm{T}^{-m} Z^{m}\right)=\mathrm{T}^{-m} \mathrm{~F}\left(Z^{m}\right)$. By observation (2) in the last part of the proof of Theorem 4.4, $\mathrm{L}\left(Z^{m}\right) \simeq \mathrm{T}^{m} \mathrm{~L}(N) \simeq \mathrm{T}^{m} \mathcal{F}$ in $\mathrm{D}^{b}(\mathbb{P})$. It follows from Corollary 4.6 that

$$
\mathrm{H}^{i}\left(F^{\bullet}\right)=\mathrm{H}^{i-m}\left(\mathrm{~F}\left(Z^{m}\right)\right) \simeq \bigoplus_{j \geqslant m-i} \mathbb{H}^{i-m}\left(\left(\mathrm{~T}^{m} \mathcal{F}\right)(j)\right)=\bigoplus_{j \geqslant m-i} \mathrm{H}^{i}(\mathcal{F}(j))
$$

One deduces that $F^{\bullet}$ is a minimal free resolution of $M^{\prime}:=\bigoplus_{j \geqslant m} \mathrm{H}^{0}(\mathcal{F}(j))$ in $S$-mod. 
The inclusion $M^{\prime} \hookrightarrow M$ lifts to a morphism of complexes $F^{\bullet} \rightarrow L^{\bullet}$. Since $M / M^{\prime}$ is of finite length, $\operatorname{Ext}_{S}^{i}\left(M / M^{\prime}, \omega_{S}\right)=0$ for $i \neq n+1$, hence

$$
\mathrm{H}^{i}\left(L^{\bullet \vee} \otimes_{S} \omega_{S}\right) \stackrel{\sim}{\rightarrow} \mathrm{H}^{i}\left(F^{\bullet \vee} \otimes_{S} \omega_{S}\right) \quad \text { for } i<n .
$$

One deduces a quasi-isomorphism $L^{\bullet \vee} \otimes_{S} \omega_{S} \rightarrow \tau^{<n}\left(F^{\bullet \vee} \otimes_{S} \omega_{S}\right)$, whence a quasiisomorphism $\tau^{>-n}\left(\left(F^{\bullet \vee} \otimes_{S} \omega_{S}\right)^{*}\right) \rightarrow\left(L^{\bullet \vee} \otimes_{S} \omega_{S}\right)^{*}$ and then a quasi-isomorphism

$$
\tau^{>0}\left(\mathrm{~T}^{-n}\left(\left(F^{\bullet \vee} \otimes_{S} \omega_{S}\right)^{*}\right)\right) \rightarrow \mathrm{T}^{-n}\left(\left(L^{\bullet \vee} \otimes_{S} \omega_{S}\right)^{*}\right) .
$$

It follows now, from the last part of Lemma $2.9(\mathrm{~b})$, that $\left(\sigma^{<m} I^{\bullet}\right) / F_{0}\left(\sigma^{<m} I^{\bullet}\right)$ is a contraction of $\mathrm{T}^{-n} \mathrm{G}\left(\left(L^{\bullet \vee} \otimes_{S} \omega_{S}\right)^{*}\right)$.

(b) Let $0 \rightarrow L^{\prime-n+1} \rightarrow \cdots \rightarrow L^{\prime 0} \rightarrow M^{\vee} \rightarrow 0$ be a minimal free resolution of $M^{\vee}$ in $S$-mod. As in the last part of Definition 3.2, one can construct from $L^{\bullet}$ and $L^{\prime \bullet}$ a Horrocks resolution $K^{\bullet}$ of $M$. One has an exact sequence:

$$
0 \rightarrow \mathrm{T}^{-1}\left(L^{\bullet \vee}\right) \rightarrow K^{\bullet \vee} \rightarrow L^{\prime \bullet} \rightarrow 0 \text {. }
$$

The Horrocks-Trautmann complex corresponding to $\mathcal{F}$ is obtained as follows: one considers a complex $N^{\bullet} \in \mathrm{ObC}^{b}\left(\Lambda\right.$-mod) such that $\mathrm{F}\left(N^{\bullet}\right) \simeq K^{\bullet}$ in $\mathrm{K}^{b}(\mathcal{P})$ and then one takes a minimal (left) free resolution $G^{\bullet}$ of $N^{\bullet}$ in $\Lambda$-mod. By Corollary 2.12, $G^{\bullet}$ is a contraction of $\mathrm{T}^{-n-1} \mathrm{G}\left(\left(\mathrm{F}\left(N^{\bullet}\right)^{\vee} \otimes_{S} \omega_{S}\right)^{*}\right)$ hence a contraction of $\mathrm{T}^{-n-1} \mathrm{G}\left(\left(K^{\bullet \vee} \otimes_{S} \omega_{S}\right)^{*}\right)$.

Using the exact sequence $(*)$, one gets a quasi-isomorphism:

$$
\tau^{\geqslant 2}\left(\mathrm{~T}^{-1}\left(L^{\bullet \vee} \otimes_{S} \omega_{S}\right)\right) \longrightarrow K^{\bullet \vee} \otimes_{S} \omega_{S},
$$

whence a quasi-isomorphism $\left(K^{\bullet \vee} \otimes_{S} \omega_{S}\right)^{*} \rightarrow \tau^{\leqslant-2}\left(\mathrm{~T}\left(\left(L^{\bullet \vee} \otimes_{S} \omega_{S}\right)^{*}\right)\right)$ and then a quasi-isomorphism:

$$
\mathrm{T}^{-n-1}\left(\left(K^{\bullet \vee} \otimes_{S} \omega_{S}\right)^{*}\right) \longrightarrow \tau^{\leqslant n-1}\left(\mathrm{~T}^{-n}\left(\left(L^{\bullet \vee} \otimes_{S} \omega_{S}\right)^{*}\right)\right)
$$

One deduces, now, from (a) and from the last part of Lemma 2.9(b), that the contraction $G^{\bullet}$ of $\mathrm{T}^{-n-1} \mathrm{G}\left(\left(K^{\bullet \vee} \otimes_{S} \omega_{S}\right)^{*}\right)$ is isomorphic to $F_{n-1} I^{\bullet} / F_{0} I^{\bullet}$.

\section{Appendix A. Cancellation of terms in a complex}

We work in an abelian category $\mathcal{A}$.

Definition A.1. If $X^{\bullet}$ and $Y^{\bullet}$ are complexes in $\mathcal{A}$ then, according to Eilenberg and MacLane [10], a contraction of $X^{\bullet}$ onto $Y^{\bullet}$ is a triple $(f, g, h)$, where $f: X^{\bullet} \rightarrow Y^{\bullet}$, $g: Y^{\bullet} \rightarrow X^{\bullet}$ are morphisms of complexes and $h \in \operatorname{Hom}^{-1}\left(X^{\bullet}, X^{\bullet}\right)$ is a homotopy operator satisfying:

$$
\text { (i) } f g=\mathrm{id}_{Y}, \quad \text { (ii) } \quad \mathrm{id}_{X}-g f=d_{X} h+h d_{X}
$$

and the side conditions:

$$
\text { (iii) } \quad f h=0, \quad \text { (iv) } h g=0, \quad \text { (v) } h^{2}=0 .
$$

The side conditions do not restrict generality. Indeed, as remarked by Lambe and Stasheff $[\mathbf{2 0}, 2.1]$, if $(f, g, h)$ satisfies (i)-(ii), if one puts $\phi:=\mathrm{id}_{X}-g f$ and $h^{\prime}:=\phi h \phi$, then $\left(f, g, h^{\prime}\right)$ satisfies (i)-(iv) $\left(d_{X} \phi=\phi d_{X}, d_{X} h+h d_{X}=\phi\right.$, and $\left.\phi^{2}=\phi\right)$. Moreover, if $h^{\prime \prime}:=h^{\prime} d_{X} h^{\prime}$ then $\left(f, g, h^{\prime \prime}\right)$ satisfies (i)-(v) $\left(h^{\prime}=h^{\prime} \phi=\phi h^{\prime}\right.$, and $\left.\phi=d_{X} h^{\prime}+h^{\prime} d_{X}\right)$. 
Notice that (i) implies that $X^{\bullet}=\operatorname{Im} g \oplus \operatorname{Ker} f$, (iv) implies that $h$ vanishes on $\operatorname{Im} g$, (iii) implies that $h$ maps $X^{\bullet}$ into $\operatorname{Ker} f$, and (ii) implies that $h \mid \operatorname{Ker} f \rightarrow \operatorname{Ker} f$ realizes a homotopy $\operatorname{id}_{\operatorname{Ker} f} \sim 0$.

Example A.2. Let $X^{\bullet}$ be a complex in $\mathcal{A}$ and assume that, for every $p \in \mathbb{Z}$, one has a decomposition $X^{p}=V^{p} \oplus W^{p} \oplus Y^{p}$ such that the component $d_{w v}^{p}: V^{p} \rightarrow W^{p+1}$ of the differential $d_{X}^{p}: X^{p} \rightarrow X^{p+1}$ is an isomorphism. Consider, for every $p \in \mathbb{Z}$, the following morphisms:

$$
\begin{gathered}
d_{Y}^{p}=d_{y y}^{p}-d_{y v}^{p}\left(d_{w v}^{p}\right)^{-1} d_{w y}^{p}: Y^{p} \longrightarrow Y^{p+1} \\
f^{p}=\left(0,-d_{y v}^{p-1}\left(d_{w v}^{p-1}\right)^{-1}, \operatorname{id}_{Y^{p}}\right): X^{p} \longrightarrow Y^{p} \\
g^{p}=\left(-\left(d_{w v}^{p}\right)^{-1} d_{w y}^{p}, 0, \operatorname{id}_{Y^{p}}\right)^{\operatorname{trsp}}: Y^{p} \longrightarrow X^{p}
\end{gathered}
$$

and $h^{p}: X^{p} \rightarrow X^{p-1}$ defined by the $3 \times 3$ matrix whose unique non-zero entry is $\left(d_{w v}^{p-1}\right)^{-1}: W^{p} \rightarrow V^{p-1}$. Then $Y^{\bullet}:=\left(Y^{p}, d_{Y}^{p}\right)_{p \in \mathbb{Z}}$ is a complex, $f:=\left(f^{p}\right)_{p \in \mathbb{Z}}$ and $g:=\left(g^{p}\right)_{p \in \mathbb{Z}}$ are morphisms of complexes and $\left(f, g, h:=\left(h^{p}\right)_{p \in \mathbb{Z}}\right)$ is a contraction of $X^{\bullet}$ onto $Y^{\bullet}$.

Example A.3. An important particular case of the previous example is that of a splitting complex $X^{\bullet}$. This means that, $\forall p \in \mathbb{Z}, Z^{p}:=\operatorname{Ker} d_{X}^{p}$ and $B^{p}:=\operatorname{Im} d_{X}^{p-1}$ are direct summands of $X^{p}$. Choose decompositions: $X^{p}=V^{p} \oplus Z^{p}$ and $Z^{p}=B^{p} \oplus H^{p}$, hence $X^{p}=V^{p} \oplus B^{p} \oplus H^{p}$. The differential $d_{X}^{p}$ vanishes on $Z^{p}$ and maps $V^{p}$ isomorphically onto $B^{p+1}$. Let $h^{p}: X^{p} \rightarrow X^{p-1}$ be the morphism defined by the $3 \times 3$ matrix whose unique non-zero term is the inverse of $V^{p-1} \stackrel{\sim}{\rightarrow} B^{p}$. Consider the complex $H^{\bullet}:=\left(H^{p}, 0\right)_{p \in \mathbb{Z}}$. Then the projection (corresponding to the above decompositions) $\pi: X^{\bullet} \rightarrow H^{\bullet}$ and the inclusion $u: H^{\bullet} \hookrightarrow X^{\bullet}$ are morphisms of complexes and $(\pi, u, h)$ is a contraction of $X^{\bullet}$ onto $H^{\bullet}$. Notice that the composite morphisms $H^{p} \hookrightarrow Z^{p} \rightarrow \mathrm{H}^{p}\left(X^{\bullet}\right), p \in \mathbb{Z}$, define an isomorphism of complexes $H^{\bullet} \stackrel{\sim}{\rightarrow} \mathrm{H}^{\bullet}\left(X^{\bullet}\right):=$ $\left(\mathrm{H}^{p}\left(X^{\bullet}\right), 0\right)_{p \in \mathbb{Z}}$.

Remark. Conversely, if a complex $X^{\bullet}$ is homotopically equivalent to a complex $H^{\bullet}$ with $d_{H}=0$ then $X^{\bullet}$ is a splitting complex.

Indeed, consider morphisms of complexes $f: X^{\bullet} \rightarrow H^{\bullet}$ and $g: H^{\bullet} \rightarrow X^{\bullet}$ such that $g f \sim \operatorname{id}_{X}$ and $f g \sim \operatorname{id}_{H}$. Choose a homotopy operator $h \in \operatorname{Hom}^{-1}\left(H^{\bullet}, H^{\bullet}\right)$ such that $\operatorname{id}_{H}-f g=d_{H} h+h d_{H}$. Since $d_{H}=0$, it follows that $f g=\operatorname{id}_{H}$, hence $X^{\bullet} \simeq X^{\prime \bullet} \oplus$ $H^{\bullet}$, where $X^{\prime \bullet}=\operatorname{Ker} f$. Choose, now, a homotopy operator $k \in \operatorname{Hom}^{-1}\left(X^{\bullet}, X^{\bullet}\right)$ such that $\operatorname{id}_{X}-g f=d_{X} k+k d_{X}$ and $f k=0$ (see the argument of Lambe and Stasheff reproduced in A.1). Then $k$ maps $X^{\bullet}$ into $\operatorname{Ker} f=X^{\prime \bullet}$ and induces a homotopy operator $h^{\prime}$ on $X^{\prime \bullet}$ such that $\mathrm{id}_{X^{\prime}}=d_{X^{\prime}} h^{\prime}+h^{\prime} d_{X^{\prime}}$. In particular, $X^{\prime \bullet}$ is acyclic, hence $B^{\prime p}:=\operatorname{Im} d_{X^{\prime}}^{p-1}=\operatorname{Ker} d_{X^{\prime}}^{p}=: Z^{\prime p}$. Now, $d_{X^{\prime}}^{p-1} \circ h^{\prime p} \mid X^{\prime p} \rightarrow B^{\prime p}$ is a left inverse for the inclusion $Z^{\prime p} \hookrightarrow X^{\prime p}$.

The next result is known in the literature as the "Basic Perturbation Lemma". In its more practical form A.6 below, it appears implicitly in Shih [22] and explicitly in R. Brown [6] and Gugenheim [14]. Its more general variant A.4 was proved by Barnes and Lambe [2]. We include here a different proof of this variant.

Lemma A.4 (Basic Perturbation Lemma). Let $(f, g, h)$ be a contraction of a complex $X^{\bullet}$ onto a complex $Y^{\bullet}$. Let $\widehat{d}_{X}=d_{X}+d_{X}^{\prime} \in \operatorname{Hom}^{1}\left(X^{\bullet}, X^{\bullet}\right)$ be a "perturbation" 
of $d_{X}$ (which means that $\widehat{d}_{X} \circ \widehat{d}_{X}=0$, i.e., that $\widehat{X}^{\bullet}:=\left(X^{p}, \widehat{d}_{X}^{p}\right)_{p \in \mathbb{Z}}$ is a complex). If $\operatorname{id}_{X}+h d_{X}^{\prime}$ is an invertible element of the $\operatorname{ring} \operatorname{Hom}^{0}\left(X^{\bullet}, X^{\bullet}\right)$ then there exist a perturbation $\widehat{d}_{Y}=d_{Y}+d_{Y}^{\prime}$ of $d_{Y}$ and a contraction $(\widehat{f}, \widehat{g}, \widehat{h})$ of the complex $\widehat{X}^{\bullet}$ onto the complex $\widehat{Y}^{\bullet}:=\left(Y^{p}, \widehat{d}_{Y}^{p}\right)_{p \in \mathbb{Z}}$.

Proof. We consider, firstly, the particular case where there exists $\phi \in \operatorname{Hom}^{1}\left(X^{\bullet}, Y^{\bullet}\right)$ such that $d_{X}^{\prime}=g \phi$. In this case:

$$
\widehat{d}_{X} g=d_{X} g+g \phi g=g d_{Y}+g \phi g=g\left(d_{Y}+\phi g\right),
$$

hence, putting $\widehat{d}_{Y}:=d_{Y}+\phi g$, one gets that $\widehat{d}_{X} g=g \widehat{d}_{Y}$. It follows that

$$
\widehat{d}_{Y} \circ \widehat{d}_{Y}=f \circ g \circ \widehat{d}_{Y} \circ \widehat{d}_{Y}=f \circ \widehat{d}_{X} \circ \widehat{d}_{X} \circ g=0,
$$

i.e., $\widehat{d}_{Y}$ is a perturbation of $d_{Y}$.

We look, now, for a perturbation $\widehat{f}=f+f^{\prime}$ of $f$ such that:

$$
\widehat{f} g=\operatorname{id}_{Y}, \quad \operatorname{id}_{X}-g \widehat{f}=\widehat{d}_{X} h+h \widehat{d}_{X} .
$$

This system of equations is equivalent to:

$$
f^{\prime} g=0, \quad-g f^{\prime}=g \phi h
$$

(because $h g=0$ ) hence it has the solution $f^{\prime}:=-\phi h$. Now, by $(*), \widehat{f} g=\operatorname{id}_{Y}$ and $g \widehat{f}$ is an endomorphism of the complex $\widehat{X}^{\bullet}$ hence:

$$
\widehat{d}_{Y} \widehat{f}-\widehat{f}_{X}=\widehat{f} g\left(\widehat{d}_{Y} \widehat{f}-\widehat{f} \widehat{d}_{X}\right)=\widehat{f}\left(\widehat{d}_{X} g \widehat{f}-\widehat{d}_{X} g \widehat{f}\right)=0,
$$

i.e., $\widehat{f}$ is a morphism of complexes from $\widehat{X}^{\bullet}$ to $\widehat{Y}^{\bullet}$. Moreover, $\widehat{f} h=-\phi h^{2}=0$. Consequently, $(\widehat{f}, g, h)$ is a contraction of $\widehat{X}^{\bullet}$ onto $\widehat{Y}^{\bullet}$.

The general case can be reduced to the particular case we have just treated as follows: $\alpha:=\operatorname{id}_{X}+h d_{X}^{\prime} \in \operatorname{Hom}^{0}\left(X^{\bullet}, X^{\bullet}\right)$ maps isomorphically the complex $\widehat{X}^{\bullet}$ onto the complex $\tilde{X}^{\bullet}=\left(X^{p}, \tilde{d}_{X}^{p}\right)_{p \in \mathbb{Z}}$, where $\widetilde{d}_{X}:=\alpha \widehat{d}_{X} \alpha^{-1}$. Using the fact that

$$
d_{X} d_{X}^{\prime}+d_{X}^{\prime} \widehat{d}_{X}=\widehat{d}_{X} \circ \widehat{d}_{X}=0
$$

and the relation (ii) from A.1 one checks easily that:

$$
\alpha \widehat{d}_{X}=d_{X} \alpha+g f d_{X}^{\prime}
$$

hence $\widetilde{d}_{X}=\alpha \widehat{d}_{X} \alpha^{-1}=d_{X}+g f d_{X}^{\prime} \alpha^{-1}$ is a perturbation of $d_{X}$ with perturbation term $g \phi$, where $\phi=f d_{X}^{\prime} \alpha^{-1}$. It follows, from the particular case, that there exist perturbations:

$$
\widehat{d}_{Y}=d_{Y}+f d_{X}^{\prime} \alpha^{-1} g, \quad \tilde{f}=f-f d_{X}^{\prime} \alpha^{-1} h
$$

such that $(\tilde{f}, g, h)$ is a contraction of the complex $\tilde{X}^{\bullet}$ onto the complex $\widehat{Y}^{\bullet}$.

One can now take: $\widehat{f}=\widetilde{f} \alpha=\widetilde{f}$ (because $f h=0$ and $h^{2}=0$ ), $\widehat{g}=\alpha^{-1} g$ and $\widehat{h}=\alpha^{-1} h \alpha=\alpha^{-1} h$ (because $h^{2}=0$ ).

Corollary A.5. Under the hypothesis of $A .4$, let $U^{\bullet}:=\operatorname{Ker} f$. The sequence $0 \rightarrow U^{\bullet} \stackrel{u}{\longrightarrow} X^{\bullet} \stackrel{f}{\longrightarrow} Y^{\bullet} \rightarrow 0$ is split exact (because $f g=\operatorname{id}_{Y}$ ), hence $X^{\bullet} \simeq Y^{\bullet} \oplus U^{\bullet}$. Then, for the complex $\widehat{Y}^{\bullet}$ obtained in the proof of $A .4$, one has: $\widehat{X}^{\bullet} \simeq \widehat{Y}^{\bullet} \oplus U^{\bullet}$. 
Proof. Since $f\left(\operatorname{id}_{X}-g f\right)=0$, it follows that there exists a morphism of complexes $v: X^{\bullet} \rightarrow U^{\bullet}$ such that $\operatorname{id}_{X}-g f=u v$. One deduces that $v u=\operatorname{id}_{U}$ and that $v g=0$, hence the sequence of complexes $0 \rightarrow Y^{\bullet} \stackrel{g}{\longrightarrow} X^{\bullet} \stackrel{v}{\longrightarrow} U^{\bullet} \rightarrow 0$ is (split) exact.

Now, using the notation from the last part of the proof of A.4, one has:

$$
v \widetilde{d}_{X}=v d_{X}+v g \phi=v d_{X}=d_{U} v
$$

hence $v$ is a morphism of complexes: $\widetilde{X}^{\bullet} \rightarrow U^{\bullet}$. The short exact sequence of complexes:

$$
0 \rightarrow \widehat{Y}^{\bullet} \stackrel{g}{\longrightarrow} \widetilde{X}^{\bullet} \stackrel{v}{\longrightarrow} U^{\bullet} \rightarrow 0
$$

is split exact (because $\widetilde{f} g=\operatorname{id}_{\widehat{Y}}$ ), hence $\widetilde{X}^{\bullet} \simeq \widehat{Y}^{\bullet} \oplus U^{\bullet}$. But one has an isomorphism of complexes $\alpha: \widehat{X}^{\bullet} \stackrel{\sim}{\rightarrow} \widetilde{X}^{\bullet}$.

Remark A.6 (The classical variant). In practice, one checks that $\mathrm{id}_{X}+h d_{X}^{\prime}$ is invertible by verifying that $h d_{X}^{\prime}$ is locally nilpotent, i.e., that $\bigcup_{i \geqslant 1} \operatorname{Ker}\left(h d_{X}^{\prime}\right)^{i}=X^{\bullet}$. In this case, the inverse of $\operatorname{id}_{X}+h d_{X}^{\prime}$ is $\operatorname{id}_{X}+\sum_{i \geqslant 1}(-1)^{i}\left(h d_{X}^{\prime}\right)^{i}$ and the proof of A.4 gives the following explicit formulae:

$$
\begin{gathered}
\widehat{d}_{Y}=d_{Y}+f d_{X}^{\prime} g+\sum_{i \geqslant 1}(-1)^{i} f d_{X}^{\prime}\left(h d_{X}^{\prime}\right)^{i} g, \quad \widehat{f}=f+\sum_{i \geqslant 1}(-1)^{i} f\left(d_{X}^{\prime} h\right)^{i} \\
\widehat{g}=g+\sum_{i \geqslant 1}(-1)^{i}\left(h d_{X}^{\prime}\right)^{i} g, \quad \widehat{h}=h+\sum_{i \geqslant 1}(-1)^{i}\left(h d_{X}^{\prime}\right)^{i} h .
\end{gathered}
$$

Finally, let us consider the case of a double complex $X^{\bullet \bullet}$ with (commuting) differentials $d_{X}^{\prime}$ and $d_{X}^{\prime \prime}$. We denote by $\mathrm{H}_{\mathrm{I}}\left(X^{\bullet \bullet}\right)$ the double complex with terms $\mathrm{H}_{\mathrm{I}}^{p q}\left(X^{\bullet \bullet}\right):=\operatorname{Ker} d_{X}^{\prime p q} / \operatorname{Im} d_{X}^{\prime p-1, q}$, with $d_{\mathrm{H}_{\mathrm{I}}}^{\prime}=0$ and with $d_{\mathrm{H}_{\mathrm{I}}}^{\prime \prime}$ induced by $d_{X}^{\prime \prime}$. We also recall the following notation: for $m \in \mathbb{Z}, \tau_{\mathrm{I}}^{\leqslant m} X^{\bullet \bullet}$ is the double subcomplex of $X^{\bullet \bullet}$ whose $(p, q)$ term is $X^{p q}$ for $p<m$, $\operatorname{Ker} d_{X}^{\prime m q}$ for $p=m$, and 0 for $p>m$. One defines, similarly, a quotient double complex $\tau_{\text {I }}^{>m} X^{\bullet \bullet}$ of $X^{\bullet \bullet}$.

The following result, which is a particular case of A.6, is stated and proved in Eisenbud et al. $[\mathbf{1 2}, 3.5]$, and it is a key technical point of that paper.

Lemma A.7. Assume that the double complex $X^{\bullet \bullet}$ satisfies the following finiteness condition: $\forall m \in \mathbb{Z}, X^{p, m-p}=0$ for $p \ll 0$. If all the rows $X^{\bullet, q}:=\left(X^{p q}, d_{X}^{p q}\right)_{p \in \mathbb{Z}}$, $q \in \mathbb{Z}$, of $X^{\bullet \bullet}$ split (see A.3) then there exists a contraction of $\operatorname{tot}\left(X^{\bullet \bullet}\right)$ onto a complex $Y^{\bullet}$, endowed with an increasing filtration $\left(F_{m} Y^{\bullet}\right)_{m \in \mathbb{Z}}$ by subcomplexes, such that:

$$
\begin{gathered}
Y^{n}=\bigoplus_{p+q=n} \mathrm{H}_{\mathrm{I}}^{p q}\left(X^{\bullet \bullet}\right), \quad \forall n \in \mathbb{Z}, \\
F_{m} Y^{n}=\bigoplus_{\substack{p+q=n \\
p \leqslant m}} \mathrm{H}_{\mathrm{I}}^{p q}\left(X^{\bullet \bullet}\right), \quad \forall m, n \in \mathbb{Z}, \\
\operatorname{gr}_{F}\left(Y^{\bullet}\right)=\operatorname{tot}\left(\mathrm{H}_{\mathrm{I}}\left(X^{\bullet \bullet}\right)\right) .
\end{gathered}
$$

Moreover, this contraction can be chosen in such a way that, for all $m \in \mathbb{Z}$, it induces a contraction of $\operatorname{tot}\left(\tau_{\mathrm{I}}^{\leqslant m} X^{\bullet \bullet}\right)$ onto $F_{m} Y^{\bullet}$ and of $\operatorname{tot}\left(\tau_{\mathrm{I}}^{>m} X^{\bullet \bullet}\right)$ onto $Y^{\bullet} / F_{m} Y^{\bullet}$. 
Proof. Recall that the differential of $\operatorname{tot}\left(X^{\bullet \bullet}\right)$ is $d_{X}^{\prime}+\delta_{X}^{\prime \prime}$, with $\delta_{X}^{\prime \prime} \mid X^{p q}:=(-1)^{p} d_{X}^{\prime \prime p q}$. Let $X_{\mathrm{I}}^{\bullet \bullet}$ be the double complex with the same terms as $X^{\bullet \bullet}$, with $d_{X_{\mathrm{I}}}^{\prime}=d_{X}^{\prime}$, and with $d_{X_{\mathrm{I}}}^{\prime \prime}=0$. A.3 provides a contraction $(\pi, u, h)$ of $\operatorname{tot}\left(X_{\mathrm{I}}^{\bullet \bullet}\right)$ onto a complex with terms $Y^{n}$ given by the formula (1) from the statement and with the differential equal to 0 . One may assume that the homotopy operator $h$ maps $X^{p q}$ into $X^{p-1, q}, \forall p, q \in \mathbb{Z}$. The differential of $\operatorname{tot}\left(X^{\bullet \bullet}\right)$ is a perturbation of $d_{X}^{\prime}$ and the finiteness condition from the statement implies that $h \delta_{X}^{\prime \prime}$ is locally nilpotent. A.6 produces now a contraction $(\widehat{\pi}, \widehat{u}, \widehat{h})$ of $\operatorname{tot}\left(X^{\bullet \bullet}\right)$ onto a complex $Y^{\bullet}$ with terms given by formula (1) from the statement and with differential:

$$
d_{Y}=\pi \delta_{X}^{\prime \prime} u+\sum_{i \geqslant 1}(-1)^{i} \pi \delta_{X}^{\prime \prime}\left(h \delta_{X}^{\prime \prime}\right)^{i} u
$$

The explicit formulae from A.6 allows one now to check easily the other assertions from the lemma.

\section{Appendix B. A comparison lemma}

Lemma B.1. Let $\mathcal{C}, \mathcal{D}$ be triangulated categories and $\Phi: \mathcal{C} \rightarrow \mathcal{D}$ an additive functor commuting with the translation functors and sending distinguished triangles to distinguished triangles. Let $X, Y$ be two objects of $\mathcal{C}$ endowed with "decreasing filtrations", i.e., with sequences of morphisms:

$$
\cdots \rightarrow F^{i+1} X \rightarrow F^{i} X \rightarrow \cdots, \quad \cdots \rightarrow F^{i+1} Y \rightarrow F^{i} Y \rightarrow \cdots
$$

such that $F^{i} X=X, F^{i} Y=Y$ for $i \ll 0$ and $F^{i} X=0, F^{i} Y=0$ for $i \gg 0$, and with the "successive quotients" replaced by distinguished triangles:

$$
F^{i+1} X \rightarrow F^{i} X \rightarrow X^{i} \rightarrow \mathrm{T} F^{i+1} X, \quad F^{i+1} Y \rightarrow F^{i} Y \rightarrow Y^{i} \rightarrow \mathrm{T} F^{i+1} Y .
$$

(a) If $\operatorname{Hom}_{\mathcal{C}}\left(X^{i}, Y^{j}\right) \rightarrow \operatorname{Hom}_{\mathcal{D}}\left(\Phi\left(X^{i}\right), \Phi\left(Y^{j}\right)\right)$ is surjective and $\operatorname{Hom}_{\mathcal{C}}\left(X^{i}, T Y^{j}\right) \rightarrow$ $\operatorname{Hom}_{\mathcal{D}}\left(\Phi\left(X^{i}\right), \Phi\left(T Y^{j}\right)\right)$ is injective, $\forall i, j$, then $\operatorname{Hom}_{\mathcal{C}}(X, Y) \rightarrow \operatorname{Hom}_{\mathcal{D}}(\Phi(X), \Phi(Y))$ is surjective.

(b) If $\operatorname{Hom}_{\mathcal{C}}\left(X^{i}, Y^{j}\right) \rightarrow \operatorname{Hom}_{\mathcal{D}}\left(\Phi\left(X^{i}\right), \Phi\left(Y^{j}\right)\right)$ is injective and $\operatorname{Hom}_{\mathcal{C}}\left(\mathrm{T} X^{i}, Y^{j}\right) \rightarrow$ $\operatorname{Hom}_{\mathcal{D}}\left(\Phi\left(\mathrm{T} X^{i}\right), \Phi\left(Y^{j}\right)\right)$ is surjective, $\forall i, j$, then $\operatorname{Hom}_{\mathcal{C}}(X, Y) \rightarrow \operatorname{Hom}_{\mathcal{D}}(\Phi(X), \Phi(Y))$ is injective.

Proof. For $p, i \in \mathbb{Z}$, we endow $\mathrm{T}^{p} F^{i} X$ with the filtration whose $j$ th term is $\mathrm{T}^{p} F^{j} X$ for $j>i$ and $\mathrm{T}^{p} F^{i} X$ for $j \leqslant i$, and similarly for $\mathrm{T}^{p} F^{i} Y$. We also endow $\mathrm{T}^{p} X^{i}$ with the filtration whose $j$ th term is $\mathrm{T}^{p} X^{i}$ for $j \leqslant i$ and 0 for $j>i$, and similarly for $\mathrm{T}^{p} Y^{i}$. We prove (a) and (b) simultaneously, by induction on $N:=\operatorname{card}\left\{i \in \mathbb{Z} \mid X^{i} \neq\right.$ $0\}+\operatorname{card}\left\{j \in \mathbb{Z} \mid Y^{j} \neq 0\right\}$. The case $N \leqslant 2$ is obvious.

For the induction step, assume, firstly, that $\operatorname{card}\left\{j \in \mathbb{Z} \mid Y^{j} \neq 0\right\} \geqslant 2$ and let $n:=$ $\inf \left\{j \in \mathbb{Z} \mid Y^{j} \neq 0\right\}$. By applying $\operatorname{Hom}_{\mathcal{C}}(X,-)$ to the complex:

$$
\mathrm{T}^{-1} Y^{n} \rightarrow F^{n+1} Y \rightarrow Y \rightarrow Y^{n} \rightarrow \mathrm{T} F^{n+1} Y
$$

and $\operatorname{Hom}_{\mathcal{D}}(\Phi(X),-)$ to the complex $\Phi((*))$, one gets a commutative diagram with exact rows and five vertical arrows. If the pair $(X, Y)$ verifies the hypothesis of (a) (resp., (b)) then $\left(X, F^{n+1} Y\right)$ and $\left(X, Y^{n}\right)$ verify the hypothesis of (a) (resp., (b)), and $\left(X, \mathrm{~T} F^{n+1} Y\right)$ verifies the hypothesis of (b) (resp., $\left(X, \mathrm{~T}^{-1} Y^{n}\right)$ verifies the hypothesis 
of (a)). Using, now, the strong form of the "Five Lemma" (see [18, Chap. I, Ex. 1.8] or $[\mathbf{1 9}, 8.3 .13])$ and taking into account the induction hypothesis, one gets the desired conclusion.

Similarly, if $\operatorname{card}\left\{i \in \mathbb{Z} \mid X^{i} \neq 0\right\} \geqslant 2$ let $m:=\inf \left\{i \in \mathbb{Z} \mid X^{i} \neq 0\right\}$. One applies $\operatorname{Hom}_{\mathcal{C}}(-, Y)$ to the complex:

$$
\mathrm{T}^{-1} X^{m} \rightarrow F^{m+1} X \rightarrow X \rightarrow X^{m} \rightarrow \mathrm{T} F^{m+1} X
$$

and $\operatorname{Hom}_{\mathcal{D}}(-, \Phi(Y))$ to the complex $\Phi\left(\left({ }^{*}\right)\right)$ and one uses again the "Five Lemma".

Before stating an useful consequence of B.1, namely Lemma B.4 below, we recall the following well known lemma.

Lemma B.2. Let $X^{\bullet}$ and $Y^{\bullet}$ be complexes in an abelian category $\mathcal{A}$ and $n \in \mathbb{Z}$.

(a) If $X^{p}=0$ (resp., $\mathrm{H}^{p}\left(X^{\bullet}\right)=0$ ) for $p>n$ then:

$$
\begin{gathered}
\operatorname{Hom}_{\mathrm{K}(\mathcal{A})}\left(X^{\bullet}, \tau^{\leqslant n} Y^{\bullet}\right) \stackrel{\sim}{\longrightarrow} \operatorname{Hom}_{\mathrm{K}(\mathcal{A})}\left(X^{\bullet}, Y^{\bullet}\right) \\
\left(\text { resp., } \operatorname{Hom}_{\mathrm{D}(\mathcal{A})}\left(X^{\bullet}, \tau^{\leqslant n} Y^{\bullet}\right) \stackrel{\sim}{\longrightarrow} \operatorname{Hom}_{\mathrm{D}(\mathcal{A})}\left(X^{\bullet}, Y^{\bullet}\right)\right) .
\end{gathered}
$$

(b) If $Y^{p}=0$ (resp., $\mathrm{H}^{p}\left(Y^{\bullet}\right)=0$ ) for $p<n$ then:

$$
\begin{gathered}
\operatorname{Hom}_{\mathrm{K}(\mathcal{A})}\left(\tau^{\geqslant n} X^{\bullet}, Y^{\bullet}\right) \stackrel{\sim}{\longrightarrow} \operatorname{Hom}_{\mathrm{K}(\mathcal{A})}\left(X^{\bullet}, Y^{\bullet}\right) \\
\left(\text { resp., } \operatorname{Hom}_{\mathrm{D}(\mathcal{A})}\left(\tau^{\geqslant n} X^{\bullet}, Y^{\bullet}\right) \stackrel{\sim}{\longrightarrow} \operatorname{Hom}_{\mathrm{D}(\mathcal{A})}\left(X^{\bullet}, Y^{\bullet}\right)\right) .
\end{gathered}
$$

Proof. The assertions about $\operatorname{Hom}_{\mathrm{K}(\mathcal{A})}$ are easy.

(a) The inverse of $\operatorname{Hom}_{\mathrm{D}(\mathcal{A})}\left(X^{\bullet}, \tau^{\leqslant n} Y^{\bullet}\right) \rightarrow \operatorname{Hom}_{\mathrm{D}(\mathcal{A})}\left(X^{\bullet}, Y^{\bullet}\right)$ associates to a morphism $X^{\bullet} \stackrel{\text { qis }}{\longleftarrow} X^{\prime \bullet} \longrightarrow Y^{\bullet}$ in $\mathrm{D}(\mathcal{A})$ the morphism $X^{\bullet} \stackrel{\text { qis }}{\longleftarrow} \tau^{\leqslant n} X^{\prime \bullet} \longrightarrow \tau^{\leqslant n} Y^{\bullet}$.

(b) The inverse of $\operatorname{Hom}_{\mathrm{D}(\mathcal{A})}\left(\tau^{\geqslant n} X^{\bullet}, Y^{\bullet}\right) \rightarrow \operatorname{Hom}_{\mathrm{D}(\mathcal{A})}\left(X^{\bullet}, Y^{\bullet}\right)$ associates to a morphism $X^{\bullet} \longrightarrow Y^{\prime \bullet} \stackrel{\text { qis }}{\longleftarrow} Y^{\bullet}$ the morphism $\tau^{\geqslant n} X^{\bullet} \longrightarrow \tau^{\geqslant n} Y^{\bullet \bullet} \stackrel{\text { qis }}{\longleftarrow} Y^{\bullet}$.

Definition B.3. If $X, Y$ are objects of an abelian category $\mathcal{A}$ and $p \in \mathbb{Z}$ then, by definition, $\operatorname{Ext}_{\mathcal{A}}^{p}(X, Y):=\operatorname{Hom}_{\mathrm{D}(\mathcal{A})}\left(X, \mathrm{~T}^{p} Y\right)$. It follows from B.2 that $\operatorname{Ext}_{\mathcal{A}}^{p}(X, Y)=$ 0 for $p<0$. Moreover, using the arguments from the proof of B.2, one sees easily that $\operatorname{Hom}_{\mathcal{A}}(X, Y) \stackrel{\sim}{\rightarrow} \operatorname{Ext}_{\mathcal{A}}^{0}(X, Y)$.

The following lemma appears, in weaker variants, in several papers like Kapranov [17] or Canonaco [7, A.5.3]. In the more precise form B.4 below, it was proved in $[\mathbf{9}, 3.3]$, under the assumption that the abelian category $\mathcal{A}$ contains sufficiently many injective objects. Here we drop this assumption using an argument similar to that used by Canonaco (this argument actually appears in the proof of B.1).

Lemma B.4. Let $\mathcal{A}$ be an abelian category, $X^{\bullet} \in \mathrm{ObC}^{-}(\mathcal{A})$ and $Y^{\bullet} \in \mathrm{ObC}^{+}(\mathcal{A})$. Consider the canonical morphism $\phi: \operatorname{Hom}_{\mathrm{K}(\mathcal{A})}\left(X^{\bullet}, Y^{\bullet}\right) \rightarrow \operatorname{Hom}_{\mathrm{D}(\mathcal{A})}\left(X^{\bullet}, Y^{\bullet}\right)$.

(a) If $\operatorname{Ext}_{\mathcal{A}}^{p-q}\left(X^{p}, Y^{q}\right)=0, \forall p>q$, then $\phi$ is surjective.

(b) If $\operatorname{Ext}_{\mathcal{A}}^{p-q-1}\left(X^{p}, Y^{q}\right)=0, \forall p>q+1$, then $\phi$ is injective.

Proof. Let $m:=\sup \left\{p \in \mathbb{Z} \mid X^{p} \neq 0\right\}$ and $n:=\inf \left\{q \in \mathbb{Z} \mid Y^{q} \neq 0\right\}$. Taking into account B.2, one may replace $X^{\bullet}$ by $\tau^{\geqslant n} X^{\bullet}$ and $Y^{\bullet}$ by $\tau^{\leqslant m} Y^{\bullet}$, hence one may assume that $X^{\bullet}$ and $Y^{\bullet}$ are bounded complexes. 
In this case, one endows $X^{\bullet}$ with the filtration $F^{i} X^{\bullet}:=\sigma^{\geqslant i} X^{\bullet}(\sigma=$ "stupid truncation"). To the semi-split short exact sequence:

$$
0 \rightarrow \sigma^{\geqslant i+1} X^{\bullet} \rightarrow \sigma^{\geqslant i} X^{\bullet} \rightarrow \mathrm{T}^{-i} X^{i} \rightarrow 0
$$

one can associate (see, for example, $[\mathbf{8}, 2(\mathrm{ii})]$ ) a distinguished triangle in $\mathrm{K}^{b}(\mathcal{A})$ :

$$
\sigma^{\geqslant i+1} X^{\bullet} \rightarrow \sigma^{\geqslant i} X^{\bullet} \rightarrow \mathrm{T}^{-i} X^{i} \rightarrow \mathrm{T} \sigma^{\geqslant i+1} X^{\bullet}
$$

One also endows $Y^{\bullet}$ with the similar filtration. The conclusion of the lemma follows now from B.1 applied to the canonical functor $\mathrm{K}^{b}(\mathcal{A}) \rightarrow \mathrm{D}^{b}(\mathcal{A})$. The hypotheses of B.1 can be easily checked in this case because most of the Hom groups involved are zero and $\operatorname{Hom}_{\mathrm{K}(\mathcal{A})}(X, Y) \stackrel{\sim}{\rightarrow} \operatorname{Hom}_{\mathrm{D}(\mathcal{A})}(X, Y), \forall X, Y \in \mathrm{Ob} \mathcal{A}$ (see B.3).

\section{References}

[1] T. Abe and M. Yoshinaga, Splitting criterion for reflexive sheaves, Proc. Amer. Math. Soc. 136 (2008), 1887-1891.

[2] D. W. Barnes and L. A. Lambe, A fixed point approach to homological perturbation theory, Proc. Amer. Math. Soc. 112 (1991), 881-892.

[3] A. Beilinson, V. Ginzburg and W. Soergel, Koszul duality patterns in representation theory, J. Amer. Math. Soc. 9 (1996), 473-527.

[4] I. N. Bernstein, I. M. Gel'fand and S. I. Gel'fand, Algebraic bundles over $\mathbb{P}^{n}$ and problems of linear algebra, Funktsional'nyi Analiz $i$ Ego Prilozhenia 12 (1978), 66-67, English translation in: Funct. Anal. Appl. 12 (1978), 212-214.

[5] C. Bertone and M. Roggero, Splitting type, global sections and Chern classes for vector bundles on $\mathbb{P}^{N}$, arXiv:0804.2985 [math.AG].

[6] R. Brown, The twisted Eilenberg-Zilber theorem, Celebrazioni Archimedee del secolo XX, Simposio di Topologia (1964), 34-37.

[7] A. Canonaco, The Beilinson complex and canonical rings of irregular surfaces, Memoirs Amer. Math. Soc. 862 (2006).

[8] I. Coandă, On the Bernstein-Gel'fand-Gel'fand correspondence and a result of Eisenbud, Fløystad, and Schreyer, J. Math. Kyoto Univ. 43 (2003), 429-439.

[9] I. Coandă and G. Trautmann, Horrocks theory and the Bernstein-Gel'fandGel'fand correspondence, Trans. Amer. Math. Soc. 385 (2005), 1015-1031.

[10] S. Eilenberg and S. MacLane, On the group H( $\pi, n) . \mathrm{I}$, Ann. Math. 58 (1953), 55-106.

[11] D. Eisenbud, Commutative algebra with a view towards algebraic geometry, Graduate Texts in Math. 150, Springer-Verlag, 1995.

[12] D. Eisenbud, G. Fløystad and F.-O. Schreyer, Sheaf cohomology and free resolutions over exterior algebras, Trans. Amer. Math. Soc. 355 (2003), 4397-4426.

[13] S. I. Gel'fand and Yu.I. Manin, Methods of homological algebra, SpringerVerlag, 1996.

[14] V. K. A. M. Gugenheim, On the chain complex of a fibration, Illinois J. Math. 16 (1972), 398-414. 
[15] G. Horrocks, Vector bundles on the punctured spectrum of a local ring, Proc. London Math. Soc. 14 (1964), 689-713.

[16] G. Horrocks, Construction of bundles on $\mathbb{P}^{n}$, In: A. Douady and J.-L. Verdier (eds.), Les equations de Yang-Mills, Séminaire E.N.S. (1977-1978), Astérisque 71-72 (1980), Soc. Math. de France, 1980, 197-203.

[17] M. M. Kapranov, Derived category of coherent sheaves on Grassmann manifolds, Funktsional'nyi Analiz i Ego Prilozheniya 17 (1983), 78-79, English translation in: Funct. Anal. Appl. 17 (1983), 145-146.

[18] M. Kashiwara and P. Schapira, Sheaves on manifolds, Grundlehren Math. Wiss. 292, Springer-Verlag, 1990.

[19] M. Kashiwara and P. Schapira, Categories and sheaves, Grundlehren Math. Wiss. 332, Springer-Verlag, 2006.

[20] L. Lambe and J. Stasheff, Applications of perturbation theory to iterated fibrations, Manuscripta Math. 58 (1987), 363-376.

[21] J.-P. Serre, Faisceaux algébriques cohérents, Ann. Math. 61 (1955), 197-278.

[22] W. Shih, Homologie des espaces fibrés, Inst. des Hautes Études Sci. 13 (1962), 93-176.

[23] G. Trautmann, Moduli of vector bundles on $\mathbb{P}_{n}(\mathbb{C})$, Math. Ann. 237 (1978), $167-186$.

Iustin Coandă Iustin.Coanda@imar.ro

Institute of Mathematics of the Romanian Academy, P. O. Box 1-764, Bucharest, RO-014700, Romania 